\title{
Learning the Regulatory Code of Gene Expression
}

\author{
Jan Zrimec ${ }^{1}$, Filip Buric ${ }^{1}$, Mariia Kokina ${ }^{1,2}$, Victor Garcia $^{3}$ and Aleksej Zelezniak ${ }^{1,4 *}$ \\ ${ }^{1}$ Department of Biology and Biological Engineering, Chalmers University of Technology, Gothenburg, Sweden, ${ }^{2}$ Novo Nordisk \\ Foundation Center for Biosustainability, Technical University of Denmark, Kongens Lyngby, Denmark, ${ }^{3}$ School of Life Sciences \\ and Facility Management, Zurich University of Applied Sciences, Wädenswil, Switzerland, ${ }^{4}$ Science for Life Laboratory, \\ Stockholm, Sweden
}

\section{OPEN ACCESS}

Edited by:

Artur Yakimovich,

Roche, United Kingdom

Reviewed by:

James J. Cai,

Texas A\&M University, United States

Chuan Ku,

Academia Sinica, Taiwan

Luigi Russo,

University of Campania Luigi Vanvitelli,

Italy

Kai Song,

Tianjin University, China

Lei Yang,

South China Agricultural University,

China

${ }^{*}$ Correspondence:

Aleksej Zelezniak

aleksej.zelezniak@chalmers.se

Specialty section:

This article was submitted to Biological Modeling and Simulation, a section of the journal

Frontiers in Molecular Biosciences

Received: 27 February 2021

Accepted: 24 May 2021

Published: 10 June 2021

Citation:

Zrimec J, Buric F, Kokina M, Garcia V and Zelezniak $A$ (2021) Learning the Regulatory Code of Gene Expression.

Front. Mol. Biosci. 8:673363.

doi: 10.3389/fmolb.2021.673363
Data-driven machine learning is the method of choice for predicting molecular phenotypes from nucleotide sequence, modeling gene expression events including protein-DNA binding, chromatin states as well as mRNA and protein levels. Deep neural networks automatically learn informative sequence representations and interpreting them enables us to improve our understanding of the regulatory code governing gene expression. Here, we review the latest developments that apply shallow or deep learning to quantify molecular phenotypes and decode the cis-regulatory grammar from prokaryotic and eukaryotic sequencing data. Our approach is to build from the ground up, first focusing on the initiating protein-DNA interactions, then specific coding and non-coding regions, and finally on advances that combine multiple parts of the gene and mRNA regulatory structures, achieving unprecedented performance. We thus provide a quantitative view of gene expression regulation from nucleotide sequence, concluding with an information-centric overview of the central dogma of molecular biology.

Keywords: gene expression prediction, cis-regulatory grammar, gene regulatory structure, mRNA \& protein abundance, chromatin accessibility, regulatory genomics, machine learning, deep neural networks

\section{INTRODUCTION}

Genetic information is stored and encoded in genes that produce an organism's phenotype by being expressed through multiple biochemical processes into a variety of functional molecules. The central dogma of molecular biology states that genetic information flows from DNA to the phenotypically relevant proteins of an organism in a unidirectional, two-step process: the transcription of DNA into messenger RNA (mRNA) is followed by translation of mRNA into protein (Watson et al., 2008).

\footnotetext{
Abbreviations: AA, amino acid; AUC, area under the receiver operating characteristic curve; AUPRC, area under the precision recall curve; biLSTM, bidirectional long short-term memory; BunDLE-seq, binding to designed library, extracting, and sequencing; CAI, codon adaptation index; ChIP-seq, chromatin immunoprecipitation sequencing; CNN, convolutional neural network; CUB, codon usage bias; DBP, DNA-binding protein; DHS, DNase I hypersensitive site; DNase-seq, DNase I hypersensitive sites sequencing; DNN, deep neural network; dsDNA, double-stranded DNA; GM, geometric mean of precision and recall; HTS, high-throughput sequencing (technology); LR, linear regression; MARS, multivariate adaptive regression splines; MCC, Matthews correlation coefficient; ML, machine learning; mRNA, messenger RNA; NN, feedforward neural network; NuSAR, nucleotide sequence activity relationships; OLS, ordinary least squares (regression); ORF, open reading frame; PBM, protein binding microarray; PIC, (eukaryotic) preinitiation complex; PLS, partial least squares (regression); premRNA, precursor mRNA; PTM, post-translational modifications; PWM, position weight matrix; RBP, RNA-binding protein; RBS, ribosome binding site; RF, random forest; RNAP, RNA polymerase; RNN, recurrent neural network; SELEX, systematic evolution of ligands by exponential enrichment; SVM, support vector machine; SNV, single nucleotide variant; ssDNA, singlestranded DNA; TF, transcription factors; TFBS, TF binding site; TIF, transcription initiation frequency; TSS, transcription start site; UTR, untranslated region.
} 
From these molecular phenotypes, further post-translational processing and cellular metabolism shape and define the observable phenotype of the organism (Nielsen, 2017). Some of the most important processes involved in gene expression are regulated at the nucleotide sequence level, spanning the coding and non-coding regulatory regions adjacent to the gene (Watson et al., 2008; Zrimec et al., 2020). For over a decade, a key trend in the field has thus been to develop computational methods that can process nucleotide sequences and interpret the regulatory code within them, to better understand gene expression and improve quantitative predictions (Segal and Widom, 2009; Levo and Segal, 2014; Li et al., 2019a). These developments are not only important for advancing molecular biology, but have practical implications as well: they are crucial for solving problems related to human disease (Lee and Young, 2013; Zhou et al., 2018a) as well as biotechnology applications (de Jongh et al., 2020).

The key interactions that govern gene expression occur among proteins and nucleic acids. Proteins search for their active binding sites by sliding and diffusion, recognizing a particular DNA site via physicochemical interactions with the molecule (Tafvizi et al., 2011; Hammar et al., 2012). Typical binding domains of DNAbinding proteins (DBPs), such as transcription factors (TFs) and polymerases, include helix-turn-helix and zinc finger domains (Watson et al., 2008). However, besides direct protein-DNA readout with the major groove of the DNA helix, which offers base-specific hydrogen bond donors, acceptors, and nonpolar groups that can be recognized by complementary groups on the amino acid side chain, the specificities of protein-DNA interactions are defined also by indirect readout (Rohs et al., 2010; Marcovitz and Levy, 2013; Inukai et al., 2017). This comprises "weak" protein-DNA interactions that depend on base pairs that are not directly contacted by the protein and are defined by conformational and physicochemical DNA properties at the specific binding sites or in their vicinity (Rohs et al., 2009; Yang et al., 2017; Zrimec and Lapanje, 2018). On the other hand, RNA is a single stranded molecule with a softer backbone than DNA and thus has more extensive secondary and tertiary structure. RNA-binding proteins (RBPs) recognize single or double stranded RNA, three-dimensional structural features of folded RNAs, or even bind RNA nonspecifically (Re et al., 2014). In regulating translation, however, multiple conserved RNA sequence motifs have been uncovered that play a key role typically via single strand or secondary structure-recognition mechanisms (Watson et al., 2008; Leppek et al., 2018). Therefore, despite the apparent monomeric simplicity of nucleic acid sequences, the problem of extracting information from them is quite complex, as they encode a rich grammar of motif occurrences, combinations and sequential properties that needs to be correctly interpreted (Siggers and Gordân, 2014; Slattery et al., 2014; Li et al., 2019a; Nagy and Nagy, 2020).

In this regard, machine learning (ML) comprises a set of algorithms that are capable of mapping complex relationships between input and target variables in a supervised fashion. The resulting predictive/descriptive models can perform classification of discrete target variables or regression of continuous ones.
Classical algorithms, which include (multiple) linear regression (LR), support vector machines (SVMs), tree-based methods such as random forests (RFs), and feedforward neural networks (NNs) (Hastie et al., 2013; Géron, 2019), commonly referred to as "shallow" methods, have in recent years been superseded by deep neural networks (DNNs) (LeCun et al., 2015). DNNs resolve many problems inherent to the shallow methods, such as the reliance on feature engineering and selection, but come at the cost of requiring orders of magnitude more training data and computational resources (Angermueller et al., 2016; Eraslan et al., 2019a). In the current big data era, however, this is a diminishing problem. The result is that the information in nucleotide sequences can now be deciphered at unprecedented scale and quality, elucidating the regulatory grammar and greatly expanding our understanding of the underlying processes and capacity to accurately predict the outcomes of gene expression (Zhou et al., 2018a; Eraslan et al., 2019a; Zrimec et al., 2020).

In the present review, we provide an overview of the latest published developments that apply ML to nucleotide sequence data in order to understand gene expression in the most well studied model organisms, including bacteria (Escherichia coli), unicellular eukaryotes (yeast, Saccharomyces cerevisiae) and multicellular eukaryotes (human, Homo sapiens). Since these organisms represent the whole spectrum of genetic regulatory complexity, with gene densities ranging from 892 (bacteria) to six (human) genes per Mbp (Zrimec et al., 2020), the knowledge and principles presented here are generally applicable to all other organisms including insects and plants (Haberle and Stark, 2018; Wang H. et al., 2020). We specifically focus on the latest developments with deep learning and compare them to the state of the art solutions with shallow methods. By reasoning from first principles, the problem of predicting gene expression levels from nucleotide sequence data is explained from the ground up by deconstructing it into the basic regulatory processes and grammatical elements. We first focus on modeling the proteinDNA interactions important for initiating transcription, which include TF binding and nucleosome positioning. We then detail the current understanding of the regulatory grammar carried within the specific coding and non-coding regulatory regions, and its involvement in defining transcript and protein abundance. Based on these principles, we review advanced modeling approaches that use multiple different parts of the gene regulatory structure or whole nucleotide sequences, demonstrating how this increases their predictive power. Finally, by considering all the results, we provide an information-centric overview of the field, and discuss the applicative potential and future outlook of the presented modeling approaches.

\section{LEARNING THE PROTEIN-DNA INTERACTIONS INITIATING GENE EXPRESSION}

One of the key regulation strategies of gene expression is at the level of transcription initiation (Watson et al., 2008), which is also the most studied and modeled regulatory mechanism (Segal and Widom, 2009; Levo and Segal, 2014). Transcription initiation is 


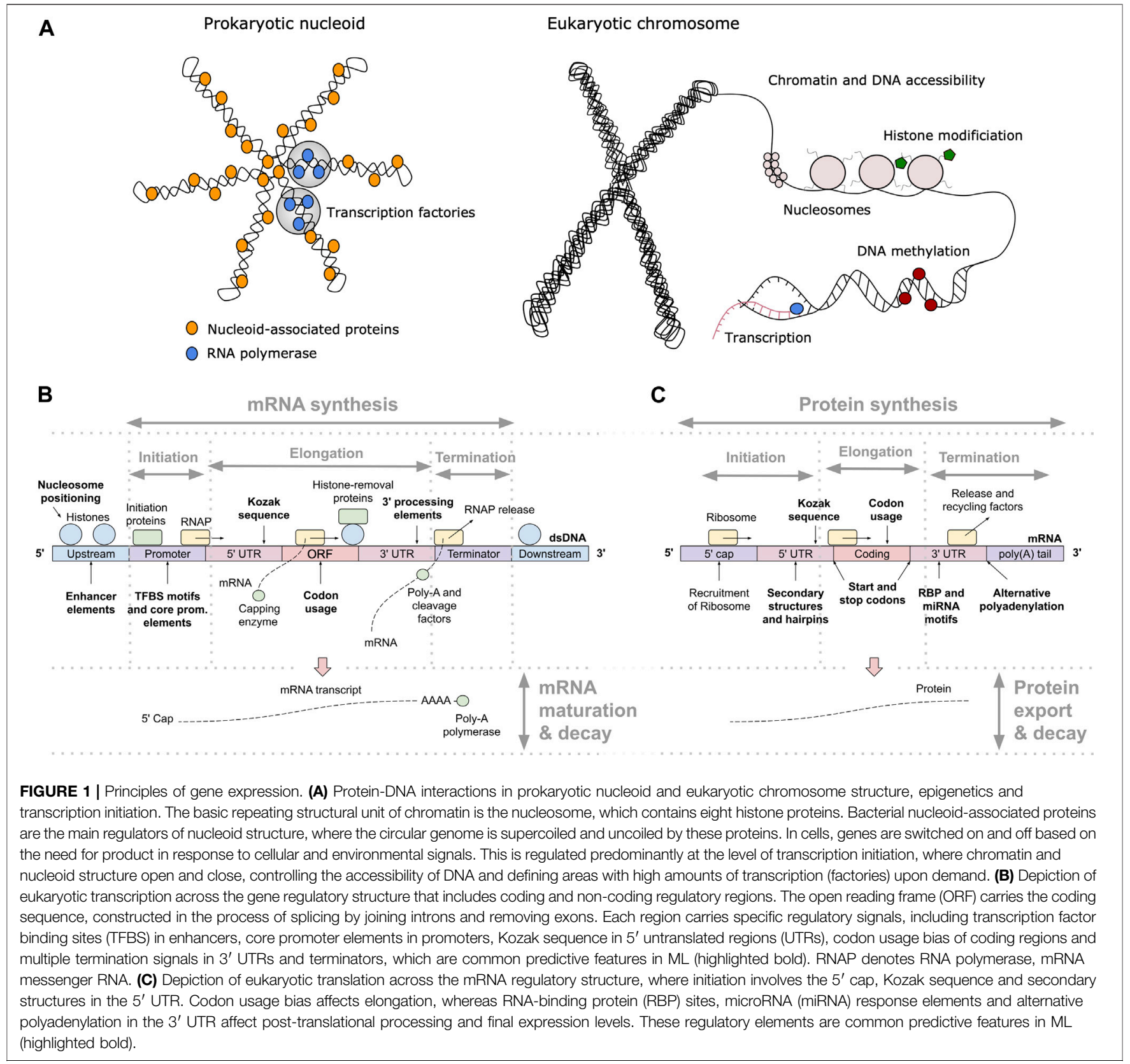

a complex process involving many different interacting DNA and protein components, including: 1 ) activating or repressing TFs that bind 6-12 bp long TF binding sites (TFBS) in enhancer and promoter regions (Watson et al., 2008) with different binding affinities and specificities (Levo and Segal, 2014), 2) nucleosomes that form around $147 \mathrm{bp}$ long DNA stretches and define chromatin accessibility, acting as general transcriptional repressors by competing with TFs for DNA binding (Segal and Widom, 2009; Struhl and Segal, 2013), 3) other components of the transcription initiation enzymatic machinery including sigma factors $(\sigma)$ in prokaryotes and components (TFIID/SAGA, mediator) of the preinitiation complex (PIC) in eukaryotes (Feklístov et al., 2014; Haberle and Stark, 2018), and 4) physicochemical and thermodynamic properties related to protein binding (Rohs et al., 2010; Inukai et al., 2017) and transcription initiation (Chen et al., 2010; Zrimec and Lapanje, 2015), such as strand dissociation around the transcription start site (TSS), giving enzymatic access to the DNA (Figure 1A). The DNA sequence preferences of nucleosomes define nucleosome organization in vivo and have been shown to account for the general depletion of nucleosomes around the starts and ends of genes as well as around TFBS, which might assist in directing TFs to their appropriate genomic sites (Segal and Widom, 2009). Apart from the DNA-guided nucleosome positioning, other epigenetic mechanisms (where functionally relevant changes to the genome do not involve a change in the nucleotide sequence), such as histone 
modification and DNA methylation, also play a vital part in transcriptional regulation (Gibney and Nolan, 2010; Miller and Grant, 2013). Together, they control the accessibility of DNA for protein binding and enzymatic processing (Watson et al., 2008) (Figure 1A). The epigenome is established and maintained by the site-specific recruitment of chromatin-modifying enzymes and their cofactors. Identifying the cis elements that regulate transcription initiation and epigenomic modification is critical for understanding the regulatory mechanisms that control gene expression patterns.

Machine learning is used to predict the locations of TFBS and their TF binding specificities, other cis-regulatory elements and binding sites, larger DNA non-coding regions such as enhancers and promoters, as well as nucleosome binding landscapes and epigenetic states. The computational tasks for inferring TFBS from DNA sequence or modeling TFBS specificity based on TF activity measurements can be framed as binary/multiclass classification and regression problems, respectively. TFBS can be predicted from the genome de novo (Jayaram et al., 2016), or analyzed based on separate measurements (Kim et al., 2007; Visel et al., 2009; Ghandi et al., 2014) or massively parallel reporter assays using highthroughput quantitative sequencing technologies (HTS), giving peak calls for various regulatory (epigenetic and transcriptional) activities across tissues and isolated cell types (Project Consortium, 2012; Roadmap Epigenomics Consortium et al., 2015). These include: 1) ChIP-seq (Chromatin immunoprecipitation sequencing) (Johnson et al., 2007) and ChIP-nexus (addition of exonuclease digestion step) (He et al., 2015) to map TF binding sites and histone modification presence, 2) DNase-seq (DNase I hypersensitive sites sequencing) (Song and Crawford, 2010) and ATAC-seq (Assay for Transposase Accessible Chromatin with high-throughput sequencing) (Buenrostro et al., 2013) to measure DNA chromatin accessibility, which typically mark nucleosomes and TF-bound sites, and 3) other methods, such as PBMs (protein binding microarrays) (Berger et al., 2006), SELEX (Systematic evolution of ligands by exponential enrichment) (Blackwell and Weintraub, 1990) and BunDLE-seq [Binding to Designed Library, Extracting, and sequencing) (Levo et al., 2015) that can provide quantitative measurements of TF binding to thousands sequences within a single experiment (further details can be found in the following publication (Barshai et al., 2020)].

Common measures for evaluating the performance of $\mathrm{ML}$ classifiers, typically on unseen data, include: 1) precision and recall, 2) the area under the receiver operating characteristic curve (AUC) that measures the tradeoff between the true positive rate (recall) and false positive rate for different thresholds, as well as 3) the area under the precision recall curve (AUPRC) that measures the tradeoff between precision and recall for different thresholds [for technical details we refer the reader to a recent review (Jiao and $\mathrm{Du}, 2016)]$. Regression models are frequently evaluated using a correlation coefficient or the coefficient of variation $\left(R^{2}\right)(\mathrm{de}$ Boer et al., 2020; Zrimec et al., 2020).

\section{Classical Machine Learning Relies on Engineered Features}

The goal of supervised ML is to learn a response function $y$ (target variable) from the set of features $x$ (explanatory variables) present in the training dataset, where $y$ describes some property related to gene expression, such as TF binding, ChIP-seq signal or mRNA abundance. With shallow learning, the DNA sequence that generally serves as the explanatory variable must be described with numerical features, such as position weight matrices (PWMs) (Stormo, 2000; Jayaram et al., 2016; Lu and Rogan, 2018), ungapped or gapped k-mer frequencies (Fletez-Brant et al., 2013; Ghandi et al., 2014; Zrimec et al., 2020), pseudo k-tuple nucleotide composition (Lin et al., 2014; Chen et al., 2015) or physicochemical and conformational (structural) properties (Rohs et al., 2009; Meysman et al., 2012; Zrimec, 2020a). Shallow methods thus require some features and methods that can describe or interpret the DNA regulatory motifs, and then use these features or motifs to build predictors. Due to their dependence on feature engineering, the shallow model training and evaluation methodology also commonly includes feature selection on all variables, retaining only the feature sets most informative for predicting the target variable. Afterward, ML models are trained on the engineered and selected feature subsets and finally, validation is performed on a held out portion of the data to assess the model performance (Ghandi et al., 2014; Zelezniak et al., 2018; Zrimec and Lapanje, 2018) (Figure 2A).

Comparison of 26 different approaches to model and learn a protein's DNA-binding specificity based on PBMs for various mouse TFs (Weirauch et al., 2013) showed that, for most TFs examined, simple models based on mononucleotide PWMs can perform similarly to more complex models, falling short only in specific cases that represented less than $10 \%$ of the examined TFs. The best-performing motifs typically have relatively low information content, consistent with widespread degeneracy in eukaryotic TF sequence preferences. Out of multiple de novo motif discovery tools that can be used locally for creating PWMs from HTS data and for scanning them against DNA, FIMO (Grant et al., 2011) and MCast (Grant et al., 2016) were found to have the best performance in their respective classes of methods that predict individual TFBSs or identify clusters, respectively (Table 1) (Jayaram et al., 2016). In an approach termed "Catchitt" for predicting cell type-specific TFBS using ensemble classifiers (Keilwagen et al., 2019), standard PWM motifs from databases were expanded with motifs learned by de novo motif discovery from ChIP-seq and DNase-seq data using sparse local inhomogeneous mixture (Slim) models (Keilwagen and Grau, 2015), which capture short to mid-range intra-motif dependencies. Catchitt earned a shared first rank in the 2017 ENCODE-DREAM in vivo TFBS prediction challenge, achieving a median AUPRC of 0.41 on test data. Despite the success of PWM-based methods, ML approaches have been shown to achieve similar or even better results. For instance, the method "QBiC-Pred" was developed to quantitatively predict TF binding changes due to sequence variants (Martin et al., 2019), using ordinary least squares (OLS) regression and HTS data containing single nucleotide variants (SNVs). The OLS models of TF binding specificity were accurate in predicting mutational effects on TF binding in vitro and in vivo ( $R^{2}$ up to 0.95$)$, outperforming widely used PWM models as well as recently developed DNNs (Alipanahi et al., 2015) on the tested data. The problem with any ML approach using k-mers as features is that it becomes susceptible to noisy training $\mathrm{k}$-mer frequencies once $k$ becomes large. This was 


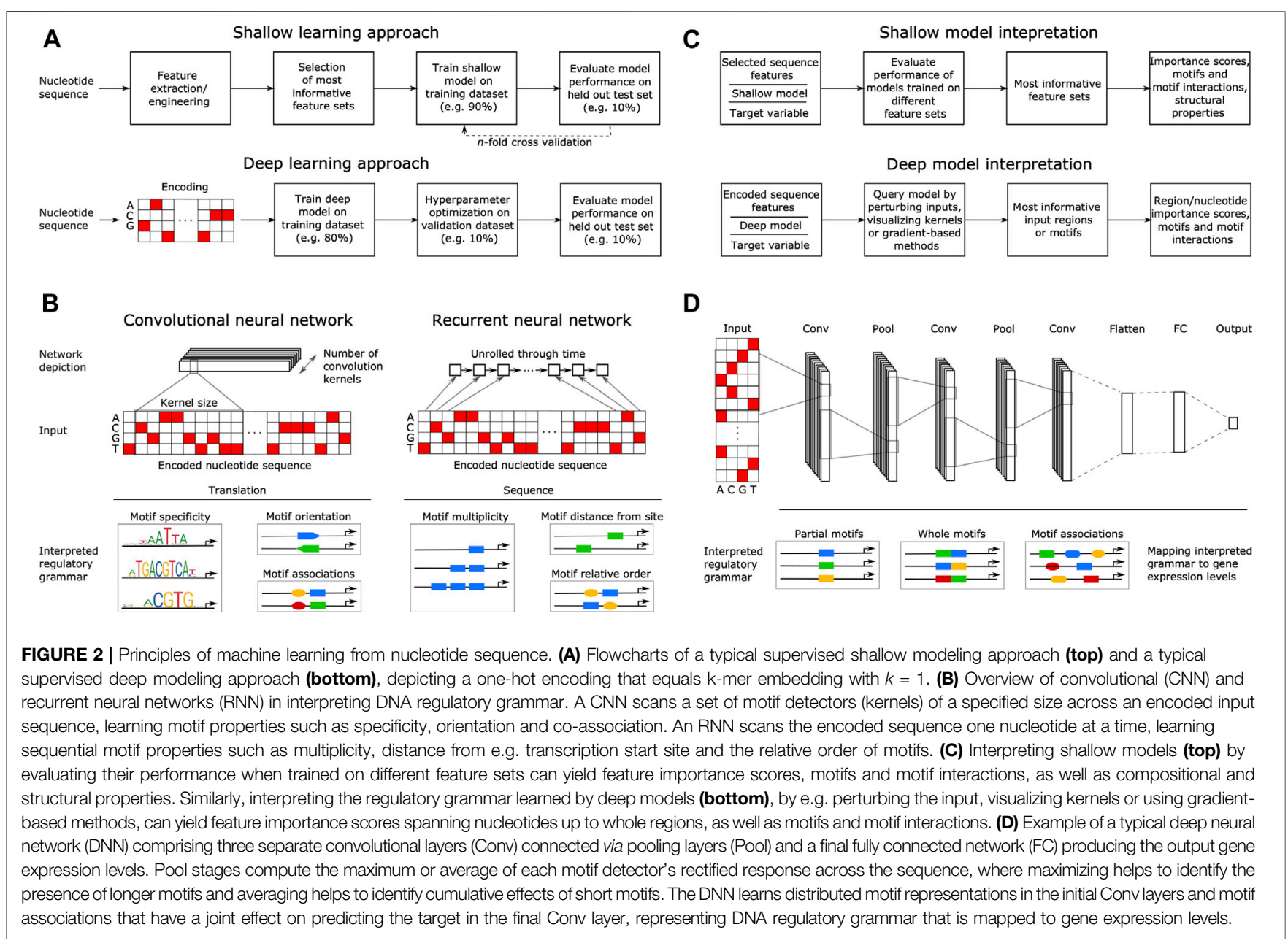

solved with methods for robust estimation of $\mathrm{k}$-mer frequencies based on alternative feature sets, where gapped k-mers were introduced as a followup to the initial k-mer method "kmerSVM" (Lee et al., 2011). The new classifier termed "gkm-SVM" predicted functional genomic regulatory elements with significantly improved accuracy compared to the original kmer-SVM, increasing the precision by up to 2 -fold and achieving an AUC of 0.97 for TFBS prediction, compared to 0.91 with kmer-SVM (Ghandi et al., 2014). In this case however, the PWM-based classifier still outperformed both methods (AUC $=0.98$ ).

In the case of epigenetic states that underlie DNA accessibility, it was shown that histone modifications can be predicted with remarkable accuracy from TF-binding profiles using LR classifiers (avg. AUC $\sim 0.86$ to 0.95 on different DNA regions in $\mathrm{H} 1$ cells), recapitulating known interactions between TFs and chromatin-modifying enzymes (Benveniste et al., 2014). This demonstrated that associations between gene expression and histone modifications do not necessarily imply a direct regulatory role for these modifications, but can be explained equally well as an indirect effect of interactions between TFs and chromatin-modifying enzymes. Similarly, a pipeline termed "Epigram" (Whitaker et al., 2015) was developed to predict histone modification and DNA methylation patterns from
DNA motifs. The authors also cataloged novel cis elements by de novo motif finding, showing that numerous motifs that have location preference and represented interactions with the sitespecific DNA-binding factors that establish and maintain epigenomic modifications. Using their method gkm-SVM (Ghandi et al., 2014) to encode cell type-specific regulatory sequence vocabularies, Lee and colleagues (Lee et al., 2015) devised a sequence-based computational method to predict the effect of regulatory variation. The effect of sequence variants was quantified by the induced change in the gkm-SVM score, "deltaSVM," which accurately predicted the impact of SNVs on DNase I hypersensitivity in their native genomes and could identify risk-conferring functional variants in validated data including autoimmune diseases, demonstrating the usefulness of this approach.

Apart from the base DNA sequence properties, structural properties have been found to improve model performance in certain cases, such as when predicting: 1) TFBS and their specificities (Abe et al., 2015; Tsai et al., 2015; Mathelier et al., 2016; Yang et al., 2017), 2) promoters and TSS sites (Meysman et al., 2012; Bansal et al., 2014; Kumar and Bansal, 2017), and 3) $\sigma$ factor binding sites (Zrimec, 2020a). These properties are directly related to protein-DNA recognition and binding (Rohs et al., 
TABLE 1 | Overview of studies modeling protein-DNA interactions that govern the initiation of gene expression from nucleotide sequence properties. Highest achieved or average scores are reported, on test sets where applicable, and include precision (prec) and recall (rec), area under the receiver operating characteristic curve (AUC), area under the precision recall curve (AUPRC), the coefficient of variation $\left(R^{2}\right)$, Pearson's correlation coefficient $(r)$, Spearman's correlation coefficient $(\rho)$ and Matthews correlation coefficient (MCC).

\begin{tabular}{|c|c|c|c|c|c|c|}
\hline Ref. & Strategy & Target var. & Explan. vars. & Method & Score & Organism \\
\hline (Jayaram et al., 2016) & Shallow & TFBS prediction & PWMs & PWM alignment algorithms & $\begin{array}{l}\text { prec }=0.73, \text { rec. } \\
=0.82\end{array}$ & Human \\
\hline (Keilwagen et al., 2019) & Shallow & TFBS prediction & $\begin{array}{l}\text { DNA motif and chromatin- } \\
\text { based features }\end{array}$ & Classifier ensembles & AUPRC $=0.81$ & Human \\
\hline (Ghandi et al., 2014) & Shallow & TFBS prediction & PWMs, gapped k-mers & SVM classification & $A \cup C=0.98$ & Human \\
\hline (Levo et al., 2015) & Shallow & TF binding specificity & $\begin{array}{l}\text { k-mers, DNA structural } \\
\text { variables }\end{array}$ & L1-regularized LR & $R^{2}=0.90$ & Yeast \\
\hline (Yang et al., 2017) & Shallow & TF binding specificity & $\begin{array}{l}\text { k-mers, DNA structural } \\
\text { variables }\end{array}$ & L2-regularized multiple LR & $R^{2}=0.90$ & Human \\
\hline (Martin et al., 2019) & Shallow & TF binding specificity & k-mers & OLS regression & $R^{2}=0.95$ & Human \\
\hline (Lin et al., 2014) & Shallow & б54 promoter prediction & $\begin{array}{l}\text { Pseudo k-tuple nucleotide } \\
\text { composition }\end{array}$ & SVM classification & $\mathrm{MCC}=0.88$ & E. coli \\
\hline (He et al., 2018) & Shallow & б70 promoter prediction & Trinucleotide-based features & SVM classification & $\mathrm{MCC}=0.92$ & E. coli \\
\hline (Benveniste et al., 2014) & Shallow & Histone modifications & k-mers, TF CHIP-seq data & LR classification & $A \cup C=0.95$ & Human \\
\hline (Whitaker et al., 2015) & Shallow & $\begin{array}{l}\text { Histone modifications, DNA } \\
\text { methylation }\end{array}$ & DNA motifs & RF classification & $A \cup C=0.96$ & Human \\
\hline (Lee et al., 2015) & Shallow & DNA chromatin accessibility & PWMs, gapped k-mers & SVM classification & $A \cup C=0.75$ & Human \\
\hline (Trabelsi et al., 2019) & Deep & TFBS prediction & k-mers & CNN + biLSTM classification & $A \cup C=0.93$ & Human \\
\hline (Zeng et al., 2016) & Deep & TFBS prediction & DNA sequence & CNN classification & $A \cup C=0.88$ & Human \\
\hline (Kelley, 2020) & Deep & TFBS prediction & DNA sequence & CNN classification & $A \cup C=0.82$ & $\begin{array}{l}\text { Human, } \\
\text { mouse }\end{array}$ \\
\hline (Chen et al., 2021) & Deep & TFBS prediction & DNA sequence & $\begin{array}{l}\text { CNN + biLSTM + attention } \\
\text { classification }\end{array}$ & $A \cup C=0.99$ & Human \\
\hline (Alipanahi et al., 2015) & Deep & TF binding specificity & DNA sequence & CNN classification & $A \cup C=0.90$ & Human \\
\hline (Wang et al., 2018) & Deep & TF binding specificity & DNA sequence & CNN regression & $\rho=0.81$ & Human \\
\hline (Avsec et al., 2021) & Deep & TF binding specificity & DNA sequence & CNN regression & $\rho=0.62$ & Human \\
\hline $\begin{array}{l}\text { (Van Brempt et al., } \\
\text { 2020) }\end{array}$ & Deep & $\begin{array}{l}\text { Transcription initiation } \\
\text { frequency }\end{array}$ & DNA sequence & CNN ordinal regression & $R^{2}=0.88$ & E. coli \\
\hline $\begin{array}{l}\text { (Zhou and Troyanskaya, } \\
\text { 2015) }\end{array}$ & Deep & $\begin{array}{l}\text { Multitask chromatin profiling } \\
\text { data }\end{array}$ & DNA sequence & CNN classification & $A \cup C=0.96$ & Human \\
\hline (Quang and Xie, 2016) & Deep & $\begin{array}{l}\text { Multitask chromatin profiling } \\
\text { data }\end{array}$ & DNA sequence & CNN + biLSTM classification & $A \cup C=0.97$ & Human \\
\hline (Park et al., 2020) & Deep & $\begin{array}{l}\text { Multitask chromatin profiling } \\
\text { data }\end{array}$ & DNA sequence & $\begin{array}{l}\text { CNN + biLSTM + attention } \\
\text { classification }\end{array}$ & $A \cup C=0.95$ & Human \\
\hline (Singh et al., 2016) & Deep & Histone modifications & DNA sequence & CNN classification & $A \cup C=0.80$ & Human \\
\hline (Singh et al., 2017) & Deep & Histone modifications & DNA sequence & $\begin{array}{l}\text { LSTM + attention } \\
\text { classification }\end{array}$ & $A \cup C=0.81$ & Human \\
\hline (Kelley et al., 2016) & Deep & DNA chromatin accessibility & DNA sequence & CNN classification & $A \cup C=0.90$ & Human \\
\hline (Kelley et al., 2018) & Deep & DNA chromatin accessibility & DNA sequence & CNN regression & $r=0.86$ & Human \\
\hline $\begin{array}{l}\text { (Angus and Eyuboglu, } \\
\text { 2018) }\end{array}$ & Deep & DNA chromatin accessibility & DNA sequence & $\mathrm{CNN}+$ attention regression & $\rho=0.59$ & Human \\
\hline $\begin{array}{l}\text { (Angermueller et al., } \\
\text { 2017) }\end{array}$ & Deep & DNA methylation & DNA sequence and features & CNN classification & $A \cup C=0.83$ & Human \\
\hline (Tian et al., 2019) & Deep & DNA methylation & DNA sequence & CNN regression & $A \cup C=0.97$ & Human \\
\hline
\end{tabular}

2009; Bishop et al., 2011; Zrimec, 2020b) and include DNA shape (Mathelier et al., 2016), thermodynamic stability (SantaLucia, 1998) and propensity for duplex destabilization (Zrimec and Lapanje, 2015), as well as flexibility and curvature related properties (Brukner et al., 1995; Geggier and Vologodskii, 2010). For instance, the dependence of TF binding specificity on the TFBS core and flanking sequence was studied using LR and BunDLE-seq data on thousands of designed sequences with single or multiple Gcn4 or Gal4 binding sites (Levo et al., 2015). By supplanting $\mathrm{k}$-mer frequencies at each position with DNA structural properties, $15 \mathrm{bp}$ flanking sequences (15 bp) of core binding sites were shown to affect the binding of TFs, as models based on combined core and flanking regions explained the highest amount of variance in the measurements $\left(R^{2}\right.$ up to 0.9 for Gal4). The contribution of DNA shape readout and its importance in core motif-flanking regions was further demonstrated using LR and HT-SELEX data across a diverse set of 215 mammalian TFs from 27 families (Yang et al., 2017), as regression models that used $\mathrm{k}$-mer and shape features generally outperformed k-mer models by $\sim 10 \%$ ( $R^{2}$ up to 0.90 ). Using feature selection techniques, positions in the TFBSs could be pinpointed where DNA shape readout is most likely to occur, and accordingly, novel DNA shape logos were proposed to visualize the DNA shape preferences of TFs. Similarly, SVM regression models of TF binding specificity based on PBM data for 68 mammalian TFs showed that shape-augmented models 
compared favorably to sequence-based models (Zhou et al., 2015), as DNA shape features reduced the dimensionality of the feature space. The authors from Rohs lab also provide an updated database of TFBS shape logos in 2020 (Chiu et al., 2020). Moreover, derivatives of DNA structural properties, such as pseudo k-tuple nucleotide compositions (Lin et al., 2014) and trinucleotide features including position-specific propensity and electron-ion potential ( $\mathrm{He}$ et al., 2018), were applied to the problem of predicting bacterial $\sigma 54$ and $\sigma 70$ promoters in E. coli, which transcribe carbon and nitrogen-related genes or regulate the transcription of most genes, respectively. The respective ML classifiers termed "iPro54-PseKNC" (Lin et al., 2014) and "70ProPred" (He et al., 2018) could accurately distinguish the specific promoters from negative examples (AUC $=0.98$ and 0.99 , respectively).

\section{Deep Neural Networks can Learn Regulatory Grammar Automatically}

In contrast to shallow architectures that are limited in their applications even when large datasets are available, deep architectures are abstracted by multiple hidden layers between $x$ and $y$. Each layer learns a new representation of the data before passing it on to the successive layers, finding hidden data structures to make accurate predictions (Mhaskar et al., 2017). The most common DNN architectures in genomics include convolutional neural networks (CNNs) and recurrent neural networks (RNNs), such as bidirectional long short-term memory (biLSTM) networks. CNNs are regularized fully connected networks that progressively scan a DNA molecule within a receptive field, where they learn to recognize the occurrence of DNA motifs (e.g. specificity, orientation and coassociation) (Eraslan et al., 2019a) (Figure 2B). Despite the capability of RNNs to learn sequential information (e.g. multiplicity, relative order), they are computationally expensive to train and certain improvements to CNNs, such as dilation (Yu and Koltun, 2015) and self-attention (Wang et al., 2017; Bello et al., 2019; Repecka et al., 2021), enable them to outperform RNNs (Gupta and Rush, 2017; Strubell et al., 2017; Trabelsi et al., 2019). Dilated convolution uses kernels with gaps to allow each kernel to capture information across a larger stretch of the input sequence, without incurring the increased cost of using RNNs (Gupta and Rush, 2017; Strubell et al., 2017). Similarly, self-attention is a special case of attention mechanism that allows kernels to focus on specific parts of the input when producing the output, allowing positions across the entire input sequence to interact and contribute to the result with different attention weights (Vaswani et al., 2017).

Deep learning does not require feature engineering or selection, since this is an inherent feature of the DNN learning process (Webb, 2018). However, it does require representing the categorical nucleotide sequence data numerically using an encoding scheme, such as one-hot, which transforms the sequence into a binary matrix with columns corresponding to each category. DNNs have thus been applied mostly on one-hot encoded nucleotide sequences as input (Eraslan et al., 2019a; Alipanahi et al., 2015), with recent reports showing that the use of k-mer embedding to represent the input sequences can improve model performance compared to one-hot encoding (itself a special case of $\mathrm{k}$-mer embedding where $k=1$ ) (Trabelsi et al., 2019). These inputs are well suited for comprehending the base DNA motif information as well as higher order interactions that describe the DNA regulatory grammar of gene expression (Eraslan et al., 2019a; Zrimec et al., 2020). Thus, DNNs achieve high predictive accuracies often surpassing those of models based on engineered features and, in our experience, using structural DNA properties does not lead to improved predictive performance with DNNs (Zrimec et al., 2020). Due to the large amount of model hyperparameters, such as network structure (e.g. number and size of kernels, Figure 2B) and training algorithm (e.g. learning rate), a special step termed hyperparameter optimization (Bergstra et al., 2015) is required for finding the best combinations of these hyperparameters and is an integral part of DNN training. To train DNNs, the data is typically split into training, validation, and testing datasets, where: 1) the model is trained on the training set by minimizing a loss function commonly MSE for regression and cross entropy for classification (Géron, 2019), 2) hyperparameter tuning is performed on the validation set and the best performing model on the validation set is chosen, and 3) the performance of the final model is evaluated on the testing set, also verifying if it overfits the data (Eraslan et al., 2019a; Zrimec et al., 2020) (Figure 2A). With DNN testing, crossvalidation is rarely performed due to the large dataset sizes and issues with algorithmic efficiency. Commonly, $10 \%$ test splits are used for testing the models trained on $80 \%$ of the data, whereas another $10 \%$ of the training data is used for the internal validation of hyperparameter selection (Géron, 2019). For further technical details we refer the reader to excellent recent reviews (Eraslan et al., 2019a; Barshai et al., 2020).

Deep methods are frequently trained on HTS peak profiles, either converted to binary scores or left continuous as a regression problem, and the underlying TFBS and specificities are interpreted by the network itself. The first such method to showcase the efficiency of DNNs for analysis of TF binding specificities was DeepBind (Alipanahi et al., 2015), where a single CNN layer was trained on sequence specificities of DNA and RNA-binding proteins as measured by several types of HTS assays (including PBM, HT-SELEX, and ChIP-seq), in a combined 12 terabases of mouse and human data. DeepBind captured binding specificities from raw sequence data by jointly discovering new motifs of hundreds of TFs along with the rules for combining them into a predictive binding score. The resulting DeepBind models could then be used to identify binding sites in test sequences and to score the effects of novel mutations, uncovering the regulatory role of disease-associated genetic variants that can affect TF binding and gene expression. Importantly, the method outperformed 14 other methods (Weirauch et al., 2013) and achieved the highest score when applied to the in vivo ChIP-seq data (avg. AUC = 0.90), suggesting that it can generalize from HT-SELEX (Jolma et al., 2013) to other data acquisition technologies despite being based on a general-purpose ML framework.

The basic approach of DeepBind was further explored and expanded upon in subsequent studies with different network layers. For instance, Zeng and co. (Zeng et al., 2016). performed 
a systematic exploration of $\mathrm{CNN}$ architectures for predicting DNA sequence binding using a similarly large set of TF data. To control potentially confounding effects, like positional or motif strength bias, they chose to explore two specific classification tasks of motif discovery (bound vs. dinucleotide shuffles per TF and cell type) and motif occupancy (bound vs. non-bound). In both tasks, classification performance increased with the number of convolution kernels (AUC up to 0.88), and the use of local pooling or additional layers had little effect on the performance. CNN architectures that took advantage of these insights exceeded the classification performance of DeepBind, emphasizing the need to use sufficient kernels to capture motif variants. With deepRAM, a tool providing an implementation of a wide selection of architectures (Trabelsi et al., 2019), it was shown that deeper, more complex architectures provide a clear advantage with sufficient training data, with hybrid $\mathrm{CNN}+\mathrm{RNN}$ architectures outperforming other methods in terms of accuracy (AUC = 0.93 with $1 \mathrm{xCNN}+$ biLSTM). However, although RNNs improve model accuracy, this comes at the expense of a loss in the interpretability of the features learned by the model. Kelley (Kelley, 2020) developed a strategy to train deep CNNs simultaneously on human and mouse genomes, which improved gene expression prediction accuracy on held out and variant sequences. Applying mouse regulatory models to analyze human genetic variants associated with molecular phenotypes and disease improved model performance (AUROC increased from 0.80 to 0.82 ), showing that the thousands of available non-human transcriptional and epigenetic profiles can be leveraged for more effective investigation of how gene regulation affects human disease. Moreover, the performance of assessing the functional impact of non-coding variants (e.g. SNVs) was further improved with DeFine (Wang et al., 2018), a regression model based on largescale TF ChIP-seq data and capable of accurately predicting real-valued TF binding intensities (Spearman's $\rho$ up to 0.81). Here, the predicted changes in the TF binding intensities between the altered sequence and the reference sequence reflected the degree of functional impact for the variant, and could accurately identify the causal functional variants from measured disease-associated variants. Similar networks have also been used in bacteria, where the online promoter design tool (ProD) (Van Brempt et al., 2020) is based on forward engineering of promoter transcription initiation frequency (TIF). By training a CNN with high-throughput DNA sequencing data from fluorescence-activated cell sorted promoter libraries of E. coli $\sigma 70$ and Bacillus subtilis $\sigma \mathrm{B}-$, $\sigma \mathrm{F}$ - and $\sigma \mathrm{W}$-dependent promoters, prediction models were capable of predicting both TIF and orthogonality of the $\sigma$-specific promoters, which facilitated development of tailored promoters, where predictions explained $\sim 88 \%$ of the variance of experimental observations.

With prediction of epigenetic states, the "DeepSEA" method (Zhou and Troyanskaya, 2015) was the first to utilize three CNN layers trained for multi-task predictions of large-scale chromatinprofiling data, including transcription factor (TF) binding, DNase I hypersensitivity sites (DHSs) and histone-mark profiles across multiple cell types. The method significantly outperformed gkm-SVM (avg. AUC of 0.96 vs. 0.90) and enabled highperformance sequence-based prediction of both DHSs (avg. AUC $=0.92$ ) and histone modifications (avg. AUC = 0.86). In the "DanQ" model (Quang and Xie, 2016) trained on similar data as DeepSEA, a hybrid CNN + RNN architecture was used in order to enhance its perception of regulatory grammar, where the CNN captured regulatory motifs and the RNN captured long-term dependencies between the motifs. The model achieved improved performance compared to DeepSEA (avg. AUC = 0.97) as well as compared to a LR baseline model, which despite its simplicity was an effective predictor (AUROC >0.70). Similarly, with histone modifications, the CNN "DeepChrome" (Singh et al., 2016) was shown to consistently outperform both SVM and RF classifiers (avg. AUC of 0.80 vs. 0.66 and 0.59, respectively). Kelley and co. (Kelley et al., 2016) introduced the open source package "Basset" that trains CNNs on a set of accessible genomic sites mapped in 164 cell types by DNaseseq, achieving improved predictive accuracy compared to previous methods, such as gkm-SVM (avg. AUC $=0.90$ vs. 0.78 ), and good overlap of SNV predictions with previous observations. Furthermore, Kelley and co. (Kelley et al., 2018) developed another $\mathrm{CNN}$, "Basenji," to predict mammalian celltype specific epigenetic and transcriptional profiles, where an unprecedented input sequence size of $131 \mathrm{kbp}$ around TSS was used, spanning distal as well as proximal regulatory elements. Indeed, model predictions regarding the influence of SNVs on gene expression were shown to align well to known variants in human populations related to disease loci (avg. Pearson's $r=0.86$ ).

To map associations between DNA sequence patterns and methylation levels at CpG-site resolution, Angermuller and co. developed "DeepCpG" (Angermueller et al., 2017). The method was evaluated on single-cell methylation data across different cell types and HTS protocols, and yielded more accurate predictions than shallow methods, such as RF (avg. AUC $=0.83$ vs. 0.80 ). The authors also showed that interpretation of the model parameters could provide insights into how sequence composition affects methylation variability. A more recent alternative approach termed "MRCNN" (Tian et al., 2019) outperformed DeepCpG (AUC up to 0.97), and de novo discovered motifs from the trained CNN kernels were shown to match known motifs.

Finally, by expanding DNN architectures with attention mechanisms to model complex dependencies among input signals, favourable results can be achieved compared to the non-attentive DNN counterparts. This was shown with multiple prediction tasks, including: 1) TFBS prediction, where "DeepGRN" (Chen et al., 2021) achieved higher unified scores in 6 of 13 targets than any of the top four methods in the 2016 ENCODE-DREAM challenge including Catchitt (Keilwagen et al., 2019), 2) histone modification, where "AttentiveChrome" (Singh et al., 2017) outperformed DeepChrome (Singh et al., 2016) in 50 out of 56 human cell types (avg. AUC of 0.81 vs. 0.80 ), 3) DNA chromatin accessibility, where the attention-based model (Angus and Eyuboglu, 2018) outperformed standard CNNs ( $\rho=0.59$ vs. 0.54 ) as well as dilated convolutions on specific experiments, and 4) multitask chromatin profiling data, where "TBiNet" (Park et al., 2020) outperformed DeepSea (Zhou and 
Troyanskaya, 2015) and DanQ (Quang and Xie, 2016) in the TFDNA binding prediction task (avg. AUC of 0.95 vs. 0.90 and 0.93 , respectively). This suggests that attention is an effective strategy to incorporate long-range sequence context into local predictions and particularly effective for gene-expression prediction.

\section{Interpreting Models to Retrieve the Learned Regulatory Grammar}

With shallow models, the most informative feature sets are interpreted by evaluating the performance of models trained on different feature sets (Ghandi et al., 2014; Zrimec and Lapanje, 2018; de Boer et al., 2020) (Figure 2C). This can yield feature importance scores, motifs (k-mers or PWMs, depending on the provided input features, Figure 2A) and motif interactions (Ghandi et al., 2014; Keilwagen and Grau, 2015), as well as compositional and structural properties (Lin et al., 2014; Yang et al., 2017), all of which comprise a compendium of regulatory grammar, informative for understanding the regulation of gene expression. Due to the inherent capability of DNNs to learn predictive motif representations, rules for cooperative TF binding interactions (Avsec et al., 2021) and higher-order sequence features, such as secondary motifs and local sequence context (Zeng et al., 2016), as well as genotypic variation effects (Zhou and Troyanskaya, 2015), they represent a powerful approach to uncover the detailed cis-regulatory grammar of genomic sequences (Figure 2C) (Koo and Ploenzke, 2020a; He et al., 2020). This is achieved by interpreting the models using approaches that include: 1) CNN kernel visualization, where typically motifs in the initial layers are visualized, 2) input perturbation-based (sensitivity) analysis, which highlights the parts of a given input sequence that are most influential for the model prediction by occluding or mutating them (Alipanahi et al., 2015; Ancona et al., 2017), 3) gradient-based methods that estimate feature importance with iterative backward and forward propagations through the network (Shrikumar et al., 2017; Montavon et al., 2018; Shrikumar et al., 2018), yielding e.g. saliency maps (Simonyan et al., 2013) and 4) higher-order interactions among sequence elements, which can be assessed e.g. by using association rule analysis (Naulaerts et al., 2015; Zrimec et al., 2020), second-order perturbations (Koo et al., 2018), self-attention networks (Ullah and Ben-Hur, 2020) or by visualizing kernels in deeper layers (Maslova et al., 2020) [interested readers are referred to (Eraslan et al., 2019a; Koo and Ploenzke, 2020a)]. Moreover, attention mechanisms were recently shown to be more effective in discovering known TF-binding motifs compared to non-attentive DNNs (Park et al., 2020), as the learned attention weights correlate with informative inputs, such as DNase-Seq coverage and DNA motifs (Chen et al., 2021), and they can provide better interpretation than other established feature visualization methods, such as saliency maps (Lanchantin et al., 2016; Singh et al., 2017).

Since these are computational approaches, they extract statistical patterns that may not immediately reflect physical properties of the variables and should be treated as hypotheses that need to be further examined (Koo and Eddy, 2019). For instance, a method can point out certain motifs or associations that are important for the model in predicting the target, but how this reflects actual physicochemical interactions can be rather hard to interpret from the model alone. Nevertheless, this is an active area of research and new solutions are frequently developed (Lundberg and Lee, 2017; Chen and Capra, 2020; Koo and Ploenzke, 2020b), where rigorous testing as well as experimentally verifying predictions will highlight the most promising approaches (Ancona et al., 2017). On the other hand, an alternative trend that is arguably more appropriate than interpreting black box models is the development of inherently interpretable models (Rudin, 2019), where prior knowledge of gene expression can be built into the deep network structure itself (Ma et al., 2018; Tareen and Kinney, 2019; Liu et al., 2020). We refer interested readers to the excellent recent review by Azodi and co. (Azodi et al., 2020).

\section{REGULATORY MECHANISMS IN SPECIFIC CODING AND NON-CODING REGIONS}

Both transcription and translation comprise multiple steps that include initiation, elongation and termination (Watson et al., 2008). Transcription of protein coding genes is controlled via the gene regulatory structure, comprised of coding and cis-regulatory regions that include promoters, untranslated regions (UTRs) and terminators, and generally proceeds in the direction from the upstream $5^{\prime}$ to downstream $3^{\prime}$ end (Figure 1B). Initiation is regulated by enhancers, promoters and $5^{\prime}$ UTRs, where the transcriptional machinery including RNA polymerase (RNAP) is guided to the correct sites on the DNA. In the elongation phase, mRNA is synthesized (transcribed) from the coding sequence, and this process terminates toward the $3^{\prime}$ UTR and terminator regions carrying termination signals. Afterward, the process of mRNA decay is triggered, which occurs in eukaryotes after the mRNA strand is matured by $5^{\prime}$ capping and $3^{\prime}$ poly(A) tail extension, and precursor mRNA (pre-mRNA) transcripts are processed by the spliceosome, removing introns (non-coding regions) and joining exons (coding regions) together (Watson et al., 2008; Wilkinson et al., 2020). The rates of mRNA synthesis and decay define the actual mRNA levels in the cell that are commonly measured with RNA-Seq (Wang et al., 2009). The DNA regions involved in mRNA synthesis carry multiple regulatory motifs, with codon usage in coding regions detailing which nucleotide triplets encoding an amino acid (AA) are used at each position, contributing to the base regulatory grammar of transcription (Plotkin and Kudla, 2011; Cheng et al., 2017). As described above, the general genomic architecture, defined by binding of histones in eukaryotes (Struhl and Segal, 2013) and nucleoid-associated proteins (NAPs) in prokaryotes (Dillon and Dorman, 2010), acts as a master regulator of transcription by controlling the accessibility of DNA to proteins (Curran et al., 2014; Morse et al., 2017).

Translation also proceeds in the direction from the $5^{\prime}$ to the $3^{\prime}$ end of an mRNA (Figure 1C) and, in bacteria, occurs simultaneously with transcription in the cytoplasm of the cell, whereas in eukaryotes transcription occurs in the nucleus and translation occurs in the cytoplasm (Watson et al., 2008). 
TABLE 2 | Overview of studies modeling gene expression-related properties from separate regulatory or coding regions. Highest achieved or average scores are reported, on test sets where applicable, and include accuracy (acc), area under the receiver operating characteristic curve (AUC), area under the precision recall curve (AUPRC), the coefficient of variation $\left(R^{2}\right)$ and Pearson's correlation coefficient $(r)$.

\begin{tabular}{|c|c|c|c|c|c|c|c|}
\hline Ref. & Strategy & Region & Target var. & Explan. vars. & Method & Score & Organism \\
\hline $\begin{array}{l}\text { (Leman et al., } \\
\text { 2018) }\end{array}$ & Shallow & Coding & Splice site prediction & $\begin{array}{l}\text { Sequence and } \\
\text { PWM features }\end{array}$ & Logistic regression & $a c c=0.96 \%$ & Human \\
\hline $\begin{array}{l}\text { (Signal et al., } \\
\text { 2018) }\end{array}$ & Shallow & Coding & Branch point prediction & Sequence features & $\begin{array}{l}\text { Gradient boosting } \\
\text { classification }\end{array}$ & $A \cup C=0.94$ & Human \\
\hline $\begin{array}{l}\text { (Zhang et al., } \\
2017 \text { a) }\end{array}$ & Shallow & Coding & Branch point prediction & Sequence features & $\begin{array}{l}\text { Mixture models } \\
\text { classification }\end{array}$ & $\mathrm{AUC}=0.82$ & Human \\
\hline $\begin{array}{l}\text { (Trösemeier et al., } \\
\text { 2019) }\end{array}$ & Shallow & Coding & Protein abundance & $\begin{array}{l}\text { Codon usage } \\
\text { features }\end{array}$ & $\begin{array}{l}\text { COSEM mathematical } \\
\text { model }\end{array}$ & $\begin{array}{l}R^{2}=0.45,0.51 \\
0.37, \text { respectively }\end{array}$ & E. coli, yeast, human \\
\hline $\begin{array}{l}\text { (Ferreira et al., } \\
\text { 2020) }\end{array}$ & Shallow & Coding & Protein abundance & Codon usage & AdaBoost regression & $R^{2}=0.95$ & Yeast \\
\hline $\begin{array}{l}\text { (Tunney et al., } \\
\text { 2018) }\end{array}$ & Shallow & Coding & $\begin{array}{l}\text { Ribosome density at } \\
\text { each codon }\end{array}$ & Codon usage & NN regression & $r=0.57$ & Yeast \\
\hline $\begin{array}{l}\text { (Zuallaert et al., } \\
\text { 2018) }\end{array}$ & Deep & Coding & Splice site prediction & DNA sequence & CNN classification & AUPRC = 0.61 & Human, A. thaliana \\
\hline $\begin{array}{l}\text { (Wang et al., } \\
\text { 2019) }\end{array}$ & Deep & Coding & Splice site prediction & DNA sequence & CNN classification & $A \cup C=0.98$ & Human \\
\hline $\begin{array}{l}\text { (Jaganathan et al., } \\
\text { 2019) }\end{array}$ & Deep & Coding & Splice site prediction & DNA sequence & CNN classification & AUPRC $=0.98$ & Human \\
\hline $\begin{array}{l}\text { (Paggi and } \\
\text { Bejerano, 2018) }\end{array}$ & Deep & Coding & Branch point prediction & DNA sequence & biLSTM classification & $A \cup C=0.71$ & Human \\
\hline $\begin{array}{l}\text { (Nazari et al., } \\
\text { 2019) }\end{array}$ & Deep & Coding & Branch point prediction & DNA sequence & $\begin{array}{l}\text { biLSTM + CNN } \\
\text { classification }\end{array}$ & $A \cup C=0.81$ & Human \\
\hline (Xu et al., 2017) & Deep & Coding & $\begin{array}{l}\text { Alternative splicing } \\
\text { prediction }\end{array}$ & $\begin{array}{l}\text { Sequence and } \\
\text { epigenetic features }\end{array}$ & $\begin{array}{l}\text { Dense DNN } \\
\text { classification }\end{array}$ & AUPRC $=0.89$ & Human \\
\hline (Lee et al., 2020) & Deep & Coding & $\begin{array}{l}\text { Alternative splicing } \\
\text { prediction }\end{array}$ & $\begin{array}{l}\text { Sequence and } \\
\text { epigenetic features }\end{array}$ & RNN classification & AUPRC $=0.8$ & Human \\
\hline $\begin{array}{l}\text { (Zhang et al., } \\
\text { 2019) }\end{array}$ & Deep & Coding & $\begin{array}{l}\text { Alternative splicing } \\
\text { prediction }\end{array}$ & RNA-seq data & $\begin{array}{l}\text { Dense DNN + bayesian } \\
\text { hypothesis testing }\end{array}$ & $A \cup C=0.87$ & Human \\
\hline (Fu et al., 2020) & Deep & Coding & Protein abundance & DNA sequence & $\begin{array}{l}\text { Multilayer biLSTM } \\
\text { regression }\end{array}$ & $R^{2}=0.52$ & E. coli \\
\hline $\begin{array}{l}\text { (Fujimoto et al., } \\
\text { 2017) }\end{array}$ & Deep & Coding & Optimal codon usage & DNA sequence & $\begin{array}{l}\text { biLSTM encoder- } \\
\text { decoder }\end{array}$ & $a c c=0.97$ & E. coli \\
\hline (Yang et al., 2019) & Deep & Coding & Transcript abundance & DNA sequence & biLSTM transducer & $a c c=0.67$ & E. coli, human \\
\hline $\begin{array}{l}\text { (Grossman et al., } \\
\text { 2017) }\end{array}$ & Shallow & Enhancer & Transcript abundance & $\begin{array}{l}\text { Motifs and pairwise } \\
\text { motif interactions }\end{array}$ & L1-regularized LR & $\begin{array}{l}R^{2}=0.38 \text { (natural), } \\
0.52 \text { (synthetic) }\end{array}$ & Human \\
\hline (Lee et al., 2011) & Shallow & Enhancer & Enhancer prediction & k-mers & SVM classification & $A \cup C=0.93$ & Human \\
\hline (Min et al., 2017) & Deep & Enhancer & Enhancer prediction & DNA sequence & CNN classification & AUPRC $=0.92$ & Human \\
\hline (Cohn et al., 2018) & Deep & Enhancer & Enhancer prediction & DNA sequence & CNN classification & $A \cup C=0.92$ & $\begin{array}{l}17 \text { mammalian } \\
\text { species including } \\
\text { human }\end{array}$ \\
\hline (Niu et al., 2019) & Deep & Enhancer & Transcript abundance & DNA sequence & CNN regression & $A \cup C=0.92$ & Human \\
\hline $\begin{array}{l}\text { (Chen and Capra, } \\
\text { 2020) }\end{array}$ & Deep & Enhancer & $\begin{array}{l}\text { Multitask regulatory } \\
\text { properties }\end{array}$ & DNA sequence & $\begin{array}{l}\text { Deep residual NN } \\
\text { classification }\end{array}$ & AUPRC $=0.98$ & Human \\
\hline $\begin{array}{l}\text { (Lubliner et al., } \\
\text { 2015) }\end{array}$ & Shallow & Promoter & $\begin{array}{l}\text { Core promoter activity via } \\
\text { reporter fluorescence }\end{array}$ & k-mers & LR & $R^{2}=0.72$ & Yeast \\
\hline $\begin{array}{l}\text { (Urtecho et al., } \\
\text { 2019) }\end{array}$ & Shallow & Promoter & mRNA abundance & $\sigma$ factor binding sites & NN regression & $R^{2}=0.96$ & E. coli \\
\hline $\begin{array}{l}\text { (Einav and Phillips, } \\
\text { 2019) }\end{array}$ & Shallow & Promoter & mRNA abundance & $\sigma$ factor binding sites & Biophysical model & $R^{2}=0.91$ & E. coli \\
\hline $\begin{array}{l}\text { (de Boer et al., } \\
\text { 2020) }\end{array}$ & Shallow & Promoter & Protein abundance & $\begin{array}{l}\text { TF binding and } \\
\text { sequence features }\end{array}$ & $\begin{array}{l}\text { L2-regularized } \\
\text { multiple LR }\end{array}$ & $\begin{array}{l}R^{2}=89 \text { (natural), } \\
94 \text { (synthetic) }\end{array}$ & Yeast \\
\hline $\begin{array}{l}\text { (Hossain et al., } \\
\text { 2020) }\end{array}$ & Shallow & Promoter & mRNA abundance & $\begin{array}{l}\text { TF binding and } \\
\text { sequence features }\end{array}$ & $\begin{array}{l}\text { L1-regularized } \\
\text { multiple LR }\end{array}$ & $R^{2}=0.49$ & E. coli, yeast \\
\hline (Leiby et al., 2020) & Deep & Promoter & $\begin{array}{l}\text { Transcription initiation } \\
\text { rate }\end{array}$ & DNA sequence & CNN regression & $R^{2}=0.90$ & E. coli \\
\hline $\begin{array}{l}\text { (Kotopka and } \\
\text { Smolke, 2020) }\end{array}$ & Deep & Promoter & Protein abundance & DNA sequence & CNN regression & $R^{2}=0.79$ & Yeast \\
\hline (Dvir et al., 2013) & Shallow & $5^{\prime}$ UTR & Protein levels & $\begin{array}{l}\text { DNA sequence } \\
\text { features + k-mers }\end{array}$ & LR & $R^{2}=0.52$ & Yeast \\
\hline (Bonde et al., & Shallow & 5' UTR & Protein abundance & RBS features & RF regression & $R^{2}=0.89$ & E. coli \\
\hline
\end{tabular}


TABLE 2 | (Continued) Overview of studies modeling gene expression-related properties from separate regulatory or coding regions. Highest achieved or average scores are reported, on test sets where applicable, and include accuracy (acc), area under the receiver operating characteristic curve (AUC), area under the precision recall curve (AUPRC), the coefficient of variation $\left(R^{2}\right)$ and Pearson's correlation coefficient $(r)$.

\begin{tabular}{|c|c|c|c|c|c|c|c|}
\hline Ref. & Strategy & Region & Target var. & Explan. vars. & Method & Score & Organism \\
\hline $\begin{array}{l}\text { (Salis et al., 2009; } \\
\text { Salis, 2011) }\end{array}$ & Shallow & 5' UTR & Protein abundance & RBS features & $\begin{array}{l}\text { Thermodynamic } \\
\text { model, LR }\end{array}$ & $\begin{array}{l}R^{2}=0.54 \text { (natural), } \\
0.84 \text { (synthetic) }\end{array}$ & E. coli \\
\hline $\begin{array}{l}\text { (Espah Borujeni } \\
\text { et al., 2017) }\end{array}$ & Shallow & $5^{\prime}$ UTR & Translation initiation rate & $\begin{array}{l}\text { N-terminal mRNA } \\
\text { structures }\end{array}$ & Biophysical model, LR & $R^{2}=0.78$ & E. coli \\
\hline (Ding et al., 2018) & Shallow & $5^{\prime}$ UTR & Protein abundance & $\begin{array}{l}\text { DNA sequence } \\
\text { activity relationships }\end{array}$ & $\begin{array}{l}\text { Partial least-squares } \\
\text { (PLS) regression }\end{array}$ & $\begin{array}{l}R^{2}=0.60 \text { (natural), } \\
0.71 \text { (synthetic) }\end{array}$ & Yeast \\
\hline $\begin{array}{l}\text { (Decoene et al., } \\
\text { 2018) }\end{array}$ & Shallow & $5^{\prime}$ UTR & Translation initiation rate & $\begin{array}{l}\text { DNA sequence } \\
\text { features }\end{array}$ & PLS regression & $R^{2}=0.73$ & Yeast \\
\hline $\begin{array}{l}\text { (Cuperus et al., } \\
\text { 2017) }\end{array}$ & Deep & 5' UTR & Protein abundance & DNA sequence & CNN regression & $R^{2}=0.62$ & Yeast \\
\hline $\begin{array}{l}\text { (Sample et al., } \\
\text { 2019) }\end{array}$ & Deep & $5^{\prime}$ UTR & Mean ribosome load & DNA sequence & CNN regression & $R^{2}=0.82$ & Human \\
\hline $\begin{array}{l}\text { (Morse et al., } \\
\text { 2017) }\end{array}$ & Shallow & $\begin{array}{l}\text { 3' UTR, } \\
\text { terminator }\end{array}$ & Protein abundance & $\begin{array}{l}\text { Nucleosome } \\
\text { occupancy score }\end{array}$ & Weighted LR & $R^{2}=0.84$ & Yeast \\
\hline $\begin{array}{l}\text { (Cambray et al., } \\
\text { 2013) }\end{array}$ & Shallow & Terminator & Termination efficiency & $\begin{array}{l}\text { DNA sequence } \\
\text { features (12) }\end{array}$ & Multiple LR & $r=0.9$ & E. coli \\
\hline (Vogel et al., 2010) & Shallow & $\begin{array}{l}\text { 3' UTR, } \\
\text { terminator }\end{array}$ & mRNA abundance & k-mers & $\begin{array}{l}\text { L1-regularized logistic } \\
\text { regression }\end{array}$ & $r=0.41$ & Yeast \\
\hline $\begin{array}{l}\text { (Bogard et al., } \\
\text { 2019) }\end{array}$ & Deep & $3^{\prime}$ UTR & $\begin{array}{l}\text { Alternative } \\
\text { polyadenylation signals }\end{array}$ & DNA sequence & CNN regression & $R^{2}=0.88$ & Human \\
\hline
\end{tabular}

Prokaryotic mRNAs have a ribosome binding site (RBS) located in the $5^{\prime}$ UTR that aids recruitment of the translation machinery (Omotajo et al., 2015). In eukaryotes, mRNAs are modified at their $5^{\prime}$ and $3^{\prime}$ ends to facilitate translation by $5^{\prime}$ capping, which recruits the ribosome to the mRNA, and addition of a $3^{\prime}$ poly(A) tail, promoting higher translation by efficient recycling of ribosomes (Mayr, 2017). The key factors for initiation are ribosome recruitment to the mRNA and correct positioning over the start codon, where the presence of a Kozak sequence in the $5^{\prime}$ UTR also increases the efficiency of translation (Nakagawa et al., 2008; Hinnebusch et al., 2016). Elongation is mostly driven by codon usage, where ribosomes synthesize proteins by concatenating one AA per codon according to the genetic code (Saier, 2019). In the termination phase, release factors terminate translation in response to stop codons and the ribosomes are recycled.

\section{Open Reading Frame and Coding Region}

Alternative splicing plays a crucial role for protein diversity in eukaryotic cells and produces several mRNA molecules from a single pre-mRNA molecule with $\sim 95 \%$ of human genes (Wilkinson et al., 2020). Conversely, in yeast, $\sim 6 \%$ of genes carry introns and very few alternative splice forms exist. RNA splicing requires a mandatory set of splicing signals including: 1 ) the splice donor site (5'ss) and splice acceptor site (3'ss) that define the exon/intron junction of each intron at the $5^{\prime}$ and $3^{\prime}$ ends, respectively, and are characterized by highly conserved dinucleotides (mainly GT and AG, respectively), and 2) the branch point site, a short and degenerate motif usually located between 18 and $44 \mathrm{bp}$ upstream of 3'ss and as far as $400 \mathrm{bp}$ upstream (Mercer et al., 2015). Alterations of these signals were found to be the most frequent cause of hereditary disease (Anna and Monika, 2018). Since 5'ss and 3'ss sequences are well characterized, reliable tools dedicated to splice site predictions have emerged, such as the logistic regression-based "SPiCE" (Leman et al., 2018), trained on
395 splice-site variants of 11 human genes, which achieved an accuracy of $95.6 \%$ and correctly predicted the impact on splicing for $98.8 \%$ of variants (Table 2 ). To predict the position of splice sites on long genomic sequences, "SpliceRover" (Zuallaert et al., 2018) and "SpliceFinder" (Wang et al., 2019) were developed using CNNs, both outperforming existing splice site prediction tools. SpliceRover achieved $\sim 10 \%$ improvement over an existing SVM-based model (Sonnenburg et al., 2007) (AUPRC $=0.61$ vs. 0.54) and SpliceFinder compared favourably to both LSTM and SVM-based approaches (AUC of 0.98 vs. 0.95 and 0.93 , respectively). A deeper, 32-layer CNN termed "SpliceAI" that accurately predicts splice junctions in pre-mRNAs was developed by Jaganathan and co. (Jaganathan et al., 2019), enabling precise prediction of noncoding genetic variants that cause cryptic splicing and outperforming shallow methods (AUPRC $=0.98$ vs. 0.95 ). The study also found that splice-altering mutations are significantly enriched in patients with rare genetic disorders, causing an estimated $9-11 \%$ of pathogenic mutations. For identification of relevant branch points, the method "Branchpointer" (Signal et al., 2018) based on gradient boosting machines showed the best performance to detect the branch points upstream of constitutive and alternative 3'ss (accuracy of 99.48 and $65.84 \%$, respectively). Alternatively, for variants occurring in a branch point area, the mixture-model based "BPP" (Zhang et al., 2017a) emerged as having the best performance to predict effects on mRNA splicing, with an accuracy of $89.17 \%$. Interestingly, two deep learning methods based on bidirectional LSTMs, "LaBranchoR" (Paggi and Bejerano, 2018) and "RNABPS" (Nazari et al., 2019), both performed worse than the above shallow methods when assessed on large scale datasets (AUC of 0.71 and 0.81, respectively, vs. 0.82 with BPP using constitutive 3'ss) (Leman et al., 2020).

Further deep learning studies on alternative splicing prediction have shown that a comprehensive splicing code should include not only genomic sequence features but also 
epigenetic properties. For instance, 16 histone modifications were used with a multi-label DNN for human embryonic stem cell differentiation in an approach termed "DeepCode" (Xu et al., 2017), achieving an AUPRC up to 0.89. Lee and co. (Lee et al., 2020) built an interpretable RNN that mimics the physical layout of splicing regulation, where the chromatin context progressively changes as the RNAP moves along the guide DNA, achieving an AUPRC of over 0.8 and showing that adjacent epigenetic signals carry useful information in addition to the actual nucleotide sequence of the guide DNA strand. Finally, to enable the characterization of differential alternative splicing between biological samples based on RNA-seq datasets even with modest coverage, the approach DARTS (Zhang et al., 2019) was developed based on a DNN and a Bayesian statistical framework used to determine the statistical significance of differential splicing events in RNA-seq data across biological conditions.

The genetic code is degenerate as most AAs are coded by multiple codons, and these codons would appear in equal frequencies if use of specific codons would not amount to any change in cellular fitness. However, the unequal use of codons that decode the same AA, termed codon usage bias (CUB), cannot be explained by mutation bias alone and is generally believed to arise from selection for improved translational efficiency (Plotkin and Kudla, 2011). Due to variations in transfer RNA (tRNA) abundances, favoring the usage of codons that correspond to more abundant tRNA can lead to faster translation. Such codons are preferred or "optimal" for translation speed up (termed codon optimality) (Hershberg and Petrov, 2008). This is supported by multiple findings in both prokaryotes and eukaryotes, showing that CUB correlates with translation efficiency (protein numbers per mRNA) (Tuller et al., 2010), certain protein structural motifs and tRNA levels (Hanson and Coller, 2018), and affects mRNA translation initiation rates and elongation rates. Furthermore, CUB indices of genes, such as the codon adaptation index (CAI) (Sharp and Li, 1987; Carbone et al., 2003), tend to correlate with the genes' expression (Ghaemmaghami et al., 2003). The role of the coding region extends beyond codon usage, however. mRNA structure was found to regulate translation (Yu et al., 2019) and mRNA hairpins can obstruct translation and override the effect of codon usage bias on translation (Cambray et al., 2018).

The strong association of mRNA levels with protein expression in a variety of organisms (Schwanhäusser et al., 2011; Csárdi et al., 2015; Liu et al., 2016) indicates a more complex background process. The selection pressure for increased protein expression can manifest in changes of DNA that optimize both translation and transcription, improving protein expression and mRNA levels, respectively. Multiple lines of recent evidence corroborate this dual role of synonymous codon changes in transcription and translation, suggesting that selection is shaping codon usage not only to optimize translational efficiency, but in response to conditions imposed by the transcription machinery as well as the physical properties of mRNA (Zhou et al., 2016; Zhou et al., 2018b). For instance, in fungi, codon optimization was found to increase mRNA and protein levels in a promoter-independent manner (Zhou et al., 2016), with CUB shown to be predictive of mRNA and protein levels, affect mRNA stability (Presnyak et al., 2015) and toxicity (Mittal et al., 2018), coevolve with transcription termination (Zhou et al., 2018b) as well as be influenced by mRNA local secondary structure (Trotta, 2013). Similarly, in E. coli, CUB was found to affect mRNA stability by defining mRNA folding at the ribosomal site (Kudla et al., 2009).

Multiple modeling studies have been performed to analyze the causes and effects of CUB as well as to find ways to optimize codon usage in order to boost gene expression levels. Codon optimization is a mature field with tools readily available on most biotechnology and DNA synthesis companies' websites (e.g. www.thermofisher.com, www.genewiz.com, www. twistbioscience.com) as well as in standalone solutions (Puigbò et al., 2007; Gould et al., 2014; Rehbein et al., 2019). Most existing optimization strategies are based on biological indices, such as CAI (Sharp and Li, 1987; Puigbò et al., 2007), and use the host's preferred codons to replace less frequently occurring ones, while also adjusting the new sequences to match the natural codon distribution in order to preserve the slow translation regions that are important for protein folding (Richardson et al., 2006; Angov et al., 2008; Hershberg and Petrov, 2009; Gaspar et al., 2012). Standard codon usage metrics were shown to be highly predictive of protein abundance. For instance, an AdaBoost model trained on a number of codon usage metrics in S. cerevisiae genes coding for high-abundance proteins (top 10\%) and low-abundance proteins (lowest $10 \%$ ) was highly predictive of these extremes of protein abundance $\left(R^{2}=0.95\right)$ (Ferreira et al., 2020).

However, while explicitly modeling existing frequency-based indices has helped to engineer high-yield proteins, it is unclear what other biological features (e.g. RNA secondary structure) should be considered during codon selection for protein synthesis maximization. To address this issue, inspired by natural language processing, deep learning was recently also applied to model CUB. Fujimoto and co. (Fujimoto et al., 2017) showed that their biLSTM-based deep language model that "translates" from DNA to optimal codon sequences, is more robust than existing frequency-based methods due to its reliance on contextual information and long-range dependencies. Similarly, a biLSTM-Transducer model of codon distribution in highly expressed bacterial and human transcripts was able to predict the next codon in a genetic sequence with improved accuracy and lower perplexity on a held out set of transcripts, outperforming previous state-of-the-art frequency-based approaches (accuracy of 0.67 vs. 0.64) (Yang et al., 2019). Another deep learning-based codon optimization approach introduced the concept of codon boxes, enabling DNA sequences to be transformed into codon box sequences, while ignoring the order of bases, and thus converting the problem of codon optimization to sequence annotation of corresponding AAs with codon boxes (Fu et al., 2020). Sequences optimized by these biLSTM codon optimization models with ones optimized by Genewiz and ThermoFisher were compared using protein expression experiments in E. coli, demonstrating that the method is efficient and competitive.

Alternatively, an algorithmic approach to replacing codons by the target organism's preferred codons was developed by Trösemeier and co. (Trösemeier et al., 2019), termed "COSEM," which simulates ribosome dynamics during mRNA 
translation and informs about protein synthesis rates per mRNA in an organism and context-dependent way. Protein synthesis rates from COSEM were integrated with further relevant covariates such as translation accuracy into a protein expression score that was used for codon optimization, with further algorithmic fine-tuning implemented in their software "OCTOPOS." The protein expression score produced competitive predictions on proteomic data from prokaryotic and eukaryotic expression systems and was shown to be superior to standard methods, achieving 3-fold increases in protein yield compared to wildtype and commercially optimized sequences (Trösemeier et al., 2019). Moreover, since ribosomes do not move uniformly along mRNAs, Tunney and co. (Tunney et al., 2018) modeled the variation in translation elongation by using a shallow $\mathrm{NN}$ to predict the ribosome density at each codon as a function of its sequence neighborhood. This enabled them to study sequence features affecting translation elongation and to design synonymous variants of a protein coding sequence in budding yeast that closely tracked the predicted translation speeds across their full range in vivo, demonstrating that control of translation elongation alone is sufficient to produce large quantitative differences in protein output.

\section{Enhancer and Promoter}

Transcriptional enhancers are located upstream of the transcription start site (TSS) and regulate spatiotemporal tissue-specific gene expression patterns over long genomic distances, which is achieved through the binding of TFs to cognate motifs (Shlyueva et al., 2014). They can typically be found farther away from the TSS with increasing genomic complexity of the organism (Mora et al., 2016; Clément et al., 2018; Zicola et al., 2019), as far as a million bps in mammals (Pennacchio et al., 2013). Enhancer function and TF binding are influenced by various features, such as the chromatin state of the genomic locus, binding site affinities, activity of bound TFs as well as interactions among TFs (Shlyueva et al., 2014; Chen and Capra, 2020). The nature of how TF interactions influence enhancer function was explored in a recent systematic analysis using in vivo binding assays with 32,115 natural and synthetic enhancers (Grossman et al., 2017). The activity of enhancers that contain motifs for $\operatorname{PPAR} \gamma$, a TF that serves as a key regulator of adipogenesis, were shown to depend on varying contributions from dozens of TFs in their immediate vicinity. Importantly, different pairs of motifs followed different interaction rules, including subadditive, additive, and superadditive interactions among specific classes of TFs, with both spatially constrained and flexible grammars.

One of the key ML tasks shedding new light on DNA features affecting enhancer function is identification of enhancer regions in genomic sequences. For instance, a k-mer based SVM framework was able to accurately identify specific types of enhancers (EP300-bound) using only genomic sequence features (Lee et al., 2011), outperforming PWM-based classifiers (AUC $=0.93$ vs. 0.87). The predictive sequence features identified by the SVM classifier revealed both enriched and depleted DNA sequence elements in the enhancers, many of which were found to play a role in specifying tissue-specific or developmental-stage-specific enhancer activity, and others that operate in a general or tissue-independent manner. The first deep learning approach to facilitate the identification of enhancers, termed "DeepEnhancer" (Min et al., 2017), relied purely on DNA sequences to predict enhancers using CNNs and transfer learning to fine-tune the model on cell line-specific enhancers. The method was superior to gkm-SVM by 7\% in both AUC and AUPRC scores, and visualizing CNN kernels as sequence logos identified motifs similar to those in the JASPAR database (Khan et al., 2018). Similarly, Cohn and co. (Cohn et al., 2018). trained deep CNNs to identify enhancer sequences in 17 mammalian species using simulated sequences, in vivo binding data of single TFs and genome-wide chromatin maps of active enhancers. High classification accuracy was obtained by combining two training strategies that identified both short (1-4 bp) low-complexity motifs and TFBS motifs unique to enhancers. The performance improved when combining positive data from all species together, demonstrating how transfer of learned parameters between networks trained on different species can improve the overall performance and supporting the existence of a shared mammalian regulatory architecture. Although identification of enhancer locations across the whole genome is necessary, it can be more important to predict in which specific tissue types they will be activated and functional. The existing DNNs, though achieving great successes in the former, cannot be directly employed in tissue-specific enhancer predictions because a specific cell or tissue type only has a limited number of available enhancer samples for training. To solve this problem, Niu and co. (Niu et al., 2019) employed a transfer learning strategy, where models trained for general enhancer predictions were retrained on tissue-specific enhancer data and achieved a significantly higher performance (geometric mean of precision and recall, $\mathrm{GM}=0.81$ vs. 0.70$)$, also surpassing gkm-SVM $(\mathrm{GM}=0.53)$. Interestingly, a very small amount of retraining epochs $(\sim 20)$ were required to complete the retraining process, giving insight into the tissue-specific regulatory rewiring and suggesting that tissue specific responses are mediated by precise changes on a small subset of binding features.

Promoters are adjacent regions directly upstream, as well as a short distance downstream, of the TSS typically spanning from 50 to a couple of $100 \mathrm{bp}$ (Sharon et al., 2012; Redden and Alper, 2015). Besides TFBS and enhancers, they contain core promoters (Lubliner et al., 2015; Haberle and Stark, 2018) in eukaryotes and $\sigma$ factor binding sites (Feklístov et al., 2014) in prokaryotes, to which the RNAP is recruited and where it acts to initiate transcription. The core promoter contains several motifs with fixed positioning relative to the TSS (Haberle and Stark, 2018), including: 1) the TATA-box motif (consensus $5^{\prime}$-TATAWAW$3^{\prime}$ ), located $\sim 30$ bp upstream of TSS and conserved from yeast to humans but found only in a minority of core promoters, 2) the initiator (Inr) motif, which directly overlaps the TSS and is more abundant than the TATA-box but not universal, with differing consensus sequence among organisms, 3) the downstream promoter element (DPE) that can accompany Inr in promoters that lack a TATA-box and is positioned 
downstream of the TSS, and 4) other motifs with defined positions relative to the TSS, including TFIIB recognition elements (BREs) and downstream core elements (DCEs) in humans (Watson et al., 2008; Haberle and Stark, 2018). A comprehensive study of yeast core promoter activity and TSS locations in thousands of native and designed sequences (Lubliner et al., 2015) showed that core promoter activity is highly correlated to that of the entire promoter and is in fact predictable from the sequence variation in core promoters $\left(R^{2}\right.$ up to 0.72 ). Interestingly, orthologous core promoters across yeast species have conserved activities, with transcription initiation in highly active core promoters focused within a narrow region and location, orientation, and flanking bases critically affecting motif function. De Boer and co. (de Boer et al., 2020) recently transcended the limitations of using native and engineered sequences with insufficient scale, instead measuring the expression output of $>100$ million fully random synthetic promoter sequences in yeast. Using shallow ML they built interpretable models of transcriptional regulation that predicted 94 and $89 \%$ of the expression driven from independent test promoters and native yeast promoter fragments, respectively, with a deep model mentioned to have achieved $96 \%$. These models allowed them to characterize each TF's specificity, activity and interactions with chromatin, showing that expression level is influenced by weak regulatory interactions, which confound designed-sequence studies, further supporting that interactions between elements in regulatory regions play an important role in orchestrating gene expression. Moreover, based on promoter libraries comprising $>1,000,000$ constitutive and inducible promoters and using deep learning, Kotopka and Smolke (Kotopka and Smolke, 2020) developed accurate predictors of promoter activity $\left(R^{2}=0.79\right)$ that were used for model-guided design of large, sequence-diverse promoter sets, confirmed to be highly active in vivo.

Prokaryotic promoters are marked by $\sigma$ factor binding sites with five distinct motifs controlling transcription initiation rates by mediating RNAP recruitment: the -35 , extended $-10,-10$, and discriminator motifs recognized by $\sigma$; and the UP element recognized by other RNAP domains (Browning and Busby, 2004; Feklístov et al., 2014). The -35 (consensus $5^{\prime}$-TTGACA- $3^{\prime}$ ) and -10 motifs (consensus $5^{\prime}$-TATAAT- $3^{\prime}$ ) are the most abundant, though the extended -10 motif can supplant -35 for initiation, both of which are recognized as dsDNA, with the remaining motifs recognized as ssDNA (Feklístov et al., 2014). By building and testing a library of 10,898 $\sigma 70$ promoter variants consisting of combinations of $-35,-10$ and UP elements, spacers, and backgrounds in E. coli (Urtecho et al., 2019), the -35 and -10 sequence elements were shown to explain over $95 \%$ of the variance in promoter strength using a shallow NN. This was an improvement over using a simple log-linear statistical model, which explained $\sim 74 \%$ of the variance, likely due to capturing nonlinear interactions with the spacer, background, and UP elements. Based on the same data from Urtecho and co. (Urtecho et al., 2019), the central claim in energy matrix models of gene expression, stating that each promoter element contributes independently and additively to gene expression and contradicting experimental measurements, was tested using biophysical models (Einav and Phillips, 2019). A "multivalent" modeling framework incorporated the effect of avidity between the -35 and -10 RNAP binding sites and could successfully characterize the full suite of gene expression data $\left(R^{2}=0.91\right)$, suggesting that avidity represents a key physical principle governing RNAP-promoter interaction, with overly tight binding inhibiting gene expression. Another use of the data by Urtecho and co. (Urtecho et al., 2019) was with deep learning, where $\mathrm{CNN}$ models were trained to predict a promoter's transcription initiation rate directly from its DNA sequence without requiring expert-labeled sequence elements (Leiby et al., 2020). The model performed comparably to the above shallow models $\left(R^{2}=0.90\right)$ and corroborated the consensus -35 , -10 and discriminator motifs as key contributors to $\sigma 70$ promoter strength. Similarly, using a "Nonrepetitive Parts Calculator" to rapidly generate and experimentally characterize thousands of bacterial promoters with transcription rates that varied across an almost 1e6-fold range, a ML model was built to explain how specific interactions controlled the promoters' transcription rates, supporting that the number of -35 and -10 motif hexamer mismatches is a potent sequence determinant (Hossain et al., 2020).

\section{5' Untranslated Region}

The key known sequence elements affecting gene expression in $5^{\prime}$ UTRs are the RBS, known as the Shine-Dalgarno sequence, in prokaryotes (Omotajo et al., 2015) and the Kozak sequence in eukaryotes (Nakagawa et al., 2008). The Shine-Dalgarno sequence is a $\sim 6$ bp highly conserved sequence (consensus $5^{\prime}$ AGGAGG-3') (Shine and Dalgarno, 1975) located 3-9 bp from the start codon, which aids recruitment of the ribosome to the mRNA and has a strong effect on the translation initiation rate, thus being highly predictive of expression (Bonde et al., 2016). In order to design synthetic RBS and enable rational control over protein expression levels, the "RBS calculator" was developed a decade ago (Salis et al., 2009; Salis, 2011). Experimental validations in $E$. coli showed that the method is accurate to within a factor of 2.3 over a range of 100,000 -fold $\left(R^{2}=0.54\right.$ on natural sequences and 0.84 on synthetic ones), correctly predicting the large effects of genetic context on identical RBS sequences that result in different protein levels. The tool was further expanded in a subsequent study (Espah Borujeni et al., 2017), where the N-terminal mRNA structures that need to be unfolded by the ribosome during translation initiation were precisely determined by designing and measuring expression levels of 27 mRNAs with N-terminal coding structures with varying positioning and energetics. The folding energetics of the N-terminal mRNA structures were determined to control translation rates only when the N-terminal mRNA structure overlaps with the ribosomal footprint, which extends 13 nucleotides past the start codon. By utilizing this improved quantification of the ribosomal footprint length, their biophysical model could more accurately predict the translation rates of 495 characterized mRNAs with diverse sequences and structures $\left(R^{2}=0.78\right)$. The contribution of the Shine-Dalgarno sequence to protein expression was further comprehensively assessed and used to develop the tool 
"EMOPEC," which can modulate the expression level of any E. coli gene by changing only a few bases (Bonde et al., 2016). Measured protein levels for $91 \%$ of the designed sequences were within twofold of the desired target levels, and predictions of these levels with RF regressors wastly outperformed RBS calculator with an $R^{2}$ of 0.89 compared to 0.44 .

In eukaryotes, the nucleotide composition of the $5^{\prime}$ UTR changes across genes and species, with highly expressed genes in S. cerevisiae preferring A-rich and G-poor 5' UTRs. The Kozak sequence, which helps to initiate translation in most mRNAs and occupies the first 6-9 nucleotides upstream of the START codon AUG, thus has the consensus $5^{\prime}$-WAMAMAA- $3^{\prime}$ in yeast (Li et al., 2017a), whereas in humans this is $5^{\prime}$-GCCGCCRMC- $3^{\prime}$ (Nakagawa et al., 2008). Measurement of protein abundance in $2,0415^{\prime}$-UTR sequence variants, differing only in positions -10 to -1 , showed that in yeast, key regulatory elements, including AUG sequence context, mRNA secondary structure, nucleosome occupancy and out-of-frame upstream AUGs conjointly modulate protein levels (Dvir et al., 2013). Based on these features, a predictive model could be developed that explains two-thirds of the expression variation. Recently, however, it was shown that also nucleotides upstream of the Kozak sequence are highly important (Li et al., 2017a). Ding and co. (Ding et al., 2018) synthesized libraries of random $5^{\prime}$ UTRs of 24 nucleotides and used a mathematical model accounting for strong epistatic interactions among bases to predict protein abundance. Then, by stepwise engineering the $5^{\prime}$ UTRs according to nucleotide sequence activity relationships (NuSAR), through repeated cycles of backbone design, directed screening, and model reconstruction, the predictive accuracy of the model was improved $\left(R^{2}=0.71\right.$ vs. initial 0.60$)$, resulting in strong $5^{\prime}$ UTRs with 5 -fold higher protein abundance than the initial sequences. Similarly, a computational approach for predicting translation initiation rates, termed "yUTR calculator," was developed using partial least-squares (PLS) regression and multiple predictive features, including presence of upstream AUGs (Decoene et al., 2018). This enabled the de novo design of $5^{\prime}$ UTRs with a diverse range of desired translation efficiencies, which were confirmed in vivo. Moreover, the importance of mRNA secondary structures in $5^{\prime}$ UTRs (Leppek et al., 2018) was also confirmed by inserting hairpin RNA structures into mRNA $5^{\prime}$ UTRs, which tuned expression levels by 100 -fold by inhibiting translation (Weenink et al., 2018). This enables generating libraries with predicted expression outputs.

To facilitate deep learning of $5^{\prime}$ UTR function in yeast, a library of half a million $50 \mathrm{bp}$ random $5^{\prime}$ UTRs was constructed and their activity assayed with growth selection experiments (Cuperus et al., 2017). A CNN model was generated that could accurately predict protein levels of both random and native sequences $\left(R^{2}=0.62\right)$, and was used to evolve highly active $5^{\prime}$ UTRs that were experimentally confirmed to lead to higher protein expression rates than the starting sequences. Similarly, in human cells, polysome profiling of a library of 280,000 randomized $5^{\prime}$ UTRs was used to develop a CNN, termed "Optimus 5-Prime," that could quantitatively capture the relationship between $5^{\prime}$ UTR sequences and their associated mean ribosome load $\left(R^{2}=0.93\right.$ vs. 0.66 with $\mathrm{k}$-mer based LR) (Sample et al., 2019). Combined with a genetic algorithm, the model was used to engineer new $5^{\prime}$ UTRs that accurately directed specified levels of ribosome loading, and also enabled finding disease-associated SNVs that affect ribosome loading and may represent a molecular basis for disease.

\section{3' Untranslated Region and Terminator}

Regulatory motifs within the $3^{\prime}$ UTR and terminator region influence transcription termination, with $3^{\prime}$ UTR regulating polyadenylation, localization and stability (decay) of mRNA as well as translation efficiency (Barrett et al., 2012; Ren et al., 2017). The $3^{\prime}$ UTR contains both binding sites for regulatory proteins and microRNAs that can decrease gene expression by either inhibiting translation or directly causing mRNA degradation. It carries the A-rich 'positioning' element (consensus 5'AAWAAA- $3^{\prime}$ in yeast and $5^{\prime}$-AATAAA- $3^{\prime}$ in humans) that directs addition of several hundred adenine residues called the poly(A) tail to the end of the mRNA transcript - the poly(A) site $5^{\prime}-\mathrm{Y}(\mathrm{A}) \mathrm{n}-3^{\prime}$, the TA-rich 'efficiency' element (most frequently $5^{\prime}$ TATWTA- $3^{\prime}$ ) upstream of the positioning element and multiple T-rich sites (Guo and Sherman, 1996; Zhao et al., 1999; Curran et al., 2015). Based on these motifs, Curran and co. (Curran et al., 2015) developed a panel of short 35-70 bp synthetic terminators for modulating gene expression in yeast, the best of which resulted in a 3.7-fold increase in protein expression compared to that of the common CYC1 terminator. Further investigation of the effects of 13,000 synthetic $3^{\prime}$ end sequences on constitutive expression levels in yeast showed that the vast majority ( 90\%) of strongly affecting mutations localized to a single positive TA-rich element, similar to the efficiency element (Vogel et al., 2010). Based on the strength of this element, dependent also on the GC content of the surrounding sequence, their classification model could explain a significant amount of measured expression variability in native $3^{\prime}$ end sequences $(r=0.41)$. Moreover, similarly as with promoters (Curran et al., 2014), Morse and co. (Morse et al., 2017) showed that terminator function can be modulated on the basis of predictions of nucleosome occupancy, with LR models highly predictive of protein output based on nucleosome occupancy scores $\left(R^{2}=0.84\right)$. Designed terminators depleted of nucleosomes achieved an almost 4 -fold higher net protein output than their original counterparts, with the main mode of action through increased termination efficiency, rather than half-life increases, suggesting a role in improved mRNA maturation.

Most genes express mRNAs with alternative polyadenylation sites at their $3^{\prime}$ ends (Tian and Manley, 2017), which were found to be remarkably heterogeneous across different yeast species. The polyadenylation pattern is determined by a broad degenerate sequence as well as local sequence reliant on poly $(\mathrm{A})$ residues that can adopt secondary structures to recruit the polyadenylation machinery (Moqtaderi et al., 2013). In humans, alternative polyadenylation leads to multiple RNA isoforms derived from a single gene, and a CNN termed 'APARENT' was trained on isoform expression data from over three million reporters to infer alternative polyadenylation in synthetic and human $3^{\prime}$ UTRs (Bogard et al., 2019). APARENT was shown to recognize known sequence motifs for polyadenylation, such as the 
positioning element, and also discover new ones, enabling the authors to engineer precisely defined polyadenylation signals and study disease-related genetic variants.

Bacterial transcription termination is known to occur via two distinct mechanisms: factor-dependent or factor-independent termination. The former relies on a regulatory protein Rho at Rho-dependent terminator sequences and is responsible for $\sim 20 \%$ of termination events in E. coli (Peters et al., 2009), whereas factor-independent termination accounts for the remaining $\sim 80 \%$ of transcription termination events and occurs at defined sequence regions known as "intrinsic terminators" that contain GC-rich regions (Roberts, 2019). Cambray and co. (Cambray et al., 2013) assembled a collection of 61 natural and synthetic intrinsic terminators that encode termination efficiencies across an 800-fold dynamic range in E. coli and, by simulating RNA folding, they found that secondary structures extending beyond the core terminator stem are likely to increase terminator activity. They developed linear sequence-function models that can accurately predict termination efficiencies $(r=0.67)$, further improving their performance by excluding terminators encoding the contextconfounding structural elements $(r=0.9)$.

\section{PREDICTING TRANSCRIPT AND PROTEIN LEVELS FROM MULTIPLE REGULATORY PARTS}

The whole nucleotide sequence is involved in gene expression. When predicting the outcomes of transcription and translation, e.g. transcript and protein abundance, it is important to consider that many of the underlying steps in these processes are dependent on the outcome of the previous steps and some can occur in tandem (Watson et al., 2008) (Figures 1B,C). Each region of the gene and mRNA regulatory structures carries distinct regulatory signals that control the specific enzymatic interactions and thus encodes a significant amount of information related to mRNA (Shalem et al., 2015; Cheng et al., 2017; Cuperus et al., 2017; Zrimec et al., 2020) and protein levels (Vogel et al., 2010; Guimaraes et al., 2014; Lahtvee et al., 2017). Moreover, multiple lines of evidence support that the gene regulatory structure is a coevolving unit in both multicellular (Castillo-Davis et al., 2004; Wittkopp et al., 2004; Hahn, 2007; Wittkopp and Kalay, 2011; Arbiza et al., 2013; Naidoo et al., 2018; Washburn et al., 2019) and unicellular eukaryotes (Tirosh et al., 2009; Park et al., 2012; Chen et al., 2016; Zrimec et al., 2020), as genes display a coupling of coding and regulatory sequence evolution (Wittkopp et al., 2004; Tirosh et al., 2009; Zrimec et al., 2020) with approximately half of all functional variation found in non-coding regions (Hahn, 2007). However, although data from multiple regions was already used in prediction of mRNA and protein levels with shallow models (Vogel et al., 2010; Guimaraes et al., 2014; Lahtvee et al., 2017), predictions based on whole gene regulatory structures spanning multiple kilobases have started to emerge only recently, with the support of deep learning (Washburn et al., 2019; Zrimec et al., 2020). Accounting for multiple regions in ML models can lead to important observations, such as differentiating and quantifying the effects of separate vs. combined regions, and determining the DNA variables across the regions as well as their interactions, which affect predictions (Figure 3A).

DNNs are highly useful in learning the regulatory code of gene expression across regulatory structures. Despite hybrid CNN + RNN architectures outperforming them in terms of accuracy, CNNs work sufficiently well for this task (Yu and Koltun, 2015; Gupta and Rush, 2017; Strubell et al., 2017) and excel in learning rich higher-order sequence features that define the cis-regulatory grammar (Siggers and Gordân, 2014; Zeng et al., 2016). Systematic analyses of network properties, such as CNN kernel size, number of kernels and number of layers as well as pooling designs (pooling layers between connected $\mathrm{CNNs}$ ), have exemplified how DNNs decode the regulatory grammar in sequence-based learning tasks (Trabelsi et al., 2019; Zeng et al., 2016; Koo and Eddy, 2019) (Figure 2D). In a multilayer $\mathrm{DNN}$, the initial one to two layers capture information on single motif occurrence, with the first layer potentially learning partial motif representations. This can be useful in complicated tasks, such as learning DNA regulatory grammar, because a wider array of representations can be combinatorially constructed from partial representations to capture the rich array of biologically important sequence patterns in vivo (Siggers and Gordân, 2014; Zrimec et al., 2020). Successive layers (e.g. Third layer) then learn to recognize motif interactions (i.e. associations in predicting the target variable) across the regulatory structure (Zeng et al., 2016; Zrimec et al., 2020). The extent to which sequence motif representations are learned by first layer kernels is influenced by kernel size and pooling, which enforce a spatial information bottleneck either from the sequence to the $\mathrm{CNN}$ or between successive CNN layers, respectively. For instance, large maxpooling $(\geq 10)$ was shown to force kernels to learn whole motifs, whereas CNNs that employ a low max-pool size $(\leq 4)$ capture partial motifs (Koo and Eddy, 2019). Similarly, the size of successive convolutional kernels can also affect the ability to assemble whole motifs in deeper layers. Moreover, the number of kernels in the first layer sets a hard constraint on the number of different sequence patterns that can be detected (Koo and Eddy, 2019). Since the scope of initial characterized sequence features limits the range and complexity of grammar representations that can be built downstream, this parameter was generally found to have the greatest impact on CNN performance (Zeng et al., 2016). Therefore, in contrast to learning tasks where the main features are simple, such as occurrence of a PWM-like motif in TFBS prediction, using multiple parts of the regulatory structures requires deeper and more complex architectures that learn distributed motif representations to address the more complex sequence patterns (Koo and Eddy, 2019; Trabelsi et al., 2019) (Figure 2D).

\section{Predicting messenger RNA Levels From Nucleotide Sequence}

Despite the importance of the whole gene regulatory structure in gene expression, very few combinations of regulatory elements 


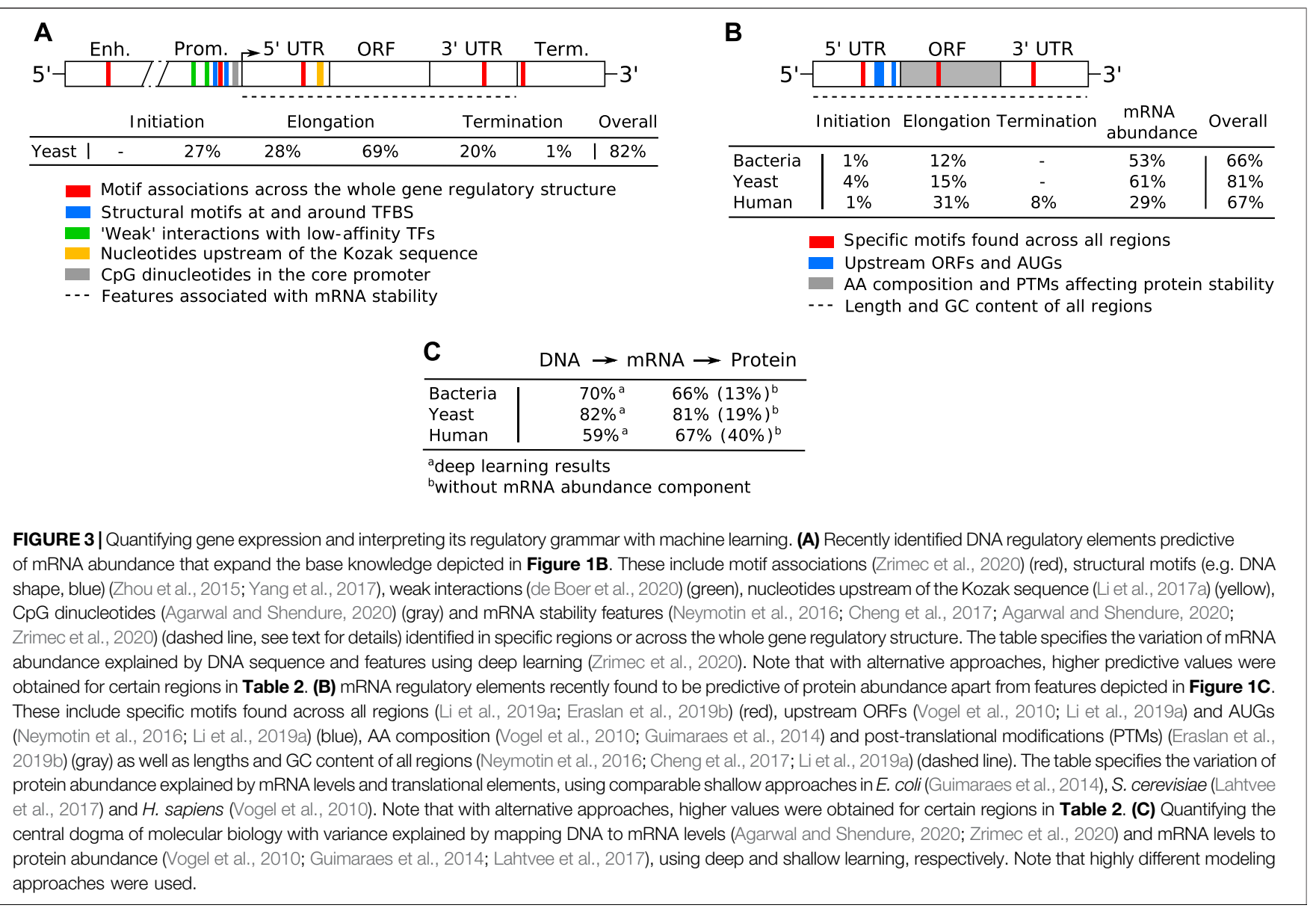

have been tested and their functional interactions remain poorly explored. To estimate the contribution of individual regulatory parts in gene expression, a combinatorial library of regulatory elements including different enhancers, core promoters, $5^{\prime}$ UTRs and transcription terminators was constructed in S. cerevisiae (Dhillon et al., 2020). A strong interaction was found between enhancers and promoters, showing that, while enhancers initiate gene expression, core promoters modulate the levels of enhancermediated expression and can positively or negatively affect expression from even the strongest enhancers. Interestingly, principal component analysis indicated that enhancer and promoter function can be explained by a single principal component. Espinar and co. (Espinar et al., 2018) tested if promoters and coding regions can be understood in isolation, or if they interact, by measuring mRNA levels for 10,000 constructs. The strength of cotranslational regulation on mRNA levels from either inducible or constitutive promoter architecture was explored using LR, where a novel mechanism for co-regulation with inducible promoters was identified (RNA helicase Dbp2), whereas with constitutive promoters, most of the information on mRNA levels was found in the coding region and not in the promoter (Table 3). Neymotin and co. (Neymotin et al., 2016) analyzed both coding regions and their interactions with other cis-regulatory variables in mRNA transcripts that affect mRNA degradations rates (which in turn affect overall mRNA abundance) using multiple LR. Multiple transcript properties were significantly associated with variation in mRNA degradation rates, including transcript length, ribosome density, CUB and GC content at the third codon position, and a model incorporating these properties explained $\sim 50 \%$ of the genome-wide variance. A similar quantitative model based on functional mRNA sequence features explained $59 \%$ of the half-life variation between genes, predicting half-life at a median relative error of $30 \%$ (Cheng et al., 2017). mRNA sequence features found to most strongly affect mRNA stability included CUB $\left(R^{2}=0.55\right)$, destabilizing $3^{\prime}$ UTR motifs, upstream AUG codons, UTR lengths and GC content.

Recently, deep learning was applied on over 20,000 mRNA datasets in seven model organisms that included bacteria, yeast and human, to examine how individual coding and non-coding regions of the gene regulatory structure interact and contribute to mRNA abundance (Zrimec et al., 2020). The CNN-based approach, termed "DeepExpression," could predict the variation of transcript levels directly from DNA sequence in all organisms, with up to 82 and $70 \%$ achieved in S. cerevisiae and E. coli, respectively, outperforming shallow methods by over $13 \%$. Apart from the DNA sequence, CUB and features associated with mRNA stability, including lengths of UTRs and open reading 
TABLE 3 | Overview of studies modeling transcript and protein-abundance related properties from combined regulatory and coding regions. Highest achieved or average scores are reported, on test sets where applicable, and include area under the receiver operating characteristic curve (AUC), area under the precision recall curve (AUPRC), the coefficient of variation $\left(R^{2}\right)$ and Spearman's correlation coefficient $(\rho)$.

\begin{tabular}{|c|c|c|c|c|c|c|c|}
\hline Ref. & Strategy & Region & Target var. & Explan. vars. & Method & Score & Organism \\
\hline (Espinar et al., 2018) & Shallow & Promoter, coding & mRNA abundance & $\begin{array}{l}\text { DNA sequence } \\
\text { features }\end{array}$ & LR & $R^{2}=0.64$ & Yeast \\
\hline $\begin{array}{l}\text { (Neymotin et al., } \\
\text { 2016) }\end{array}$ & Shallow & mRNA transcript & $\begin{array}{l}\text { mRNA stability } \\
\text { (degradation rates) }\end{array}$ & mRNA features & Multiple LR & $R^{2}=0.50$ & Yeast \\
\hline (Cheng et al., 2017) & Shallow & mRNA transcript & $\begin{array}{l}\text { mRNA stability (half- } \\
\text { life) }\end{array}$ & mRNA features & Multivariate LR & $R^{2}=0.59$ & Yeast \\
\hline (Zhou et al., 2018a) & Deep & $\begin{array}{l}\text { Whole gene } \\
\text { regulatory structure }\end{array}$ & mRNA abundance & DNA sequence & $\begin{array}{l}\mathrm{CNN}+\mathrm{L} 2- \\
\text { regularized LR }\end{array}$ & $A \cup C=0.82$ & Human \\
\hline (Zrimec et al., 2020) & Deep & $\begin{array}{l}\text { Whole gene } \\
\text { regulatory structure }\end{array}$ & mRNA abundance & $\begin{array}{l}\text { DNA sequence and } \\
\text { features }\end{array}$ & CNN regression & $\begin{array}{l}R^{2}=0.82,0.70,0.42 \\
\text { respectively }\end{array}$ & $\begin{array}{l}\text { Yeast, E. coli, } \\
\text { human }\end{array}$ \\
\hline $\begin{array}{l}\text { (Agarwal and } \\
\text { Shendure, 2020) }\end{array}$ & Deep & Promoter, coding & mRNA abundance & $\begin{array}{l}\text { DNA sequence and } \\
\text { features }\end{array}$ & CNN regression & $R^{2}=0.59$ & Human \\
\hline (Zhang et al., 2020) & Deep & $\begin{array}{l}\text { Whole gene } \\
\text { regulatory structure }\end{array}$ & mRNA abundance & DNA sequence & ResNet regression & $\rho=0.80$ & Human \\
\hline $\begin{array}{l}\text { (Guimaraes et al., } \\
\text { 2014) }\end{array}$ & Shallow & mRNA transcript & Protein abundance & mRNA features & PLS regression & $R^{2}=0.66$ & E. coli \\
\hline $\begin{array}{l}\text { (Lahtvee et al., } \\
\text { 2017) }\end{array}$ & Shallow & mRNA transcript & Protein abundance & mRNA features & $\begin{array}{l}\text { MARS nonlinear } \\
\text { regression }\end{array}$ & $R^{2}=0.81$ & Yeast \\
\hline (Vogel et al., 2010) & Shallow & mRNA transcript & Protein abundance & mRNA features & $\begin{array}{l}\text { MARS nonlinear } \\
\text { regression }\end{array}$ & $R^{2}=0.67$ & Human \\
\hline (Li et al., 2019a) & Shallow & mRNA transcript & Translation rates & mRNA features & Multivariate LR & $\begin{array}{l}R^{2}=0.81,0.42 \\
\text { respectively }\end{array}$ & Yeast, human \\
\hline $\begin{array}{l}\text { (Terai and Asai, } \\
\text { 2020) }\end{array}$ & Shallow & mRNA transcript & Protein abundance & $\begin{array}{l}\text { mRNA features of } \\
\text { translation initiation }\end{array}$ & $\mathrm{RF}$ regression & $\rho=0.76$ & E. coli \\
\hline (Li et al., 2017b) & Shallow & mRNA transcript & Translation rates & mRNA features & Bayesian model & $\begin{array}{l}R^{2}=0.20\left(T R_{\mathrm{mD}} ;\right. \\
0.80\left(\mathrm{TR}_{\mathrm{mIND}}\right)\end{array}$ & Yeast \\
\hline $\begin{array}{l}\text { (Eraslan et al., } \\
\text { 2019b) }\end{array}$ & Shallow & mRNA transcript & Protein-to-RNA ratio & $\begin{array}{l}\text { mRNA sequence and } \\
\text { features }\end{array}$ & Multivariate LR & $R^{2}=0.62$ & Human \\
\hline $\begin{array}{l}\text { (Zhang et al., } \\
\text { 2017b) }\end{array}$ & Deep & mRNA transcript & $\begin{array}{l}\text { Translation initiation } \\
\text { sites }\end{array}$ & mRNA sequence & $\begin{array}{l}\text { CNN + RNN } \\
\text { classification }\end{array}$ & AUPRC = 0.62 & Human \\
\hline (Zhang et al., 2017c) & Deep & mRNA transcript & $\begin{array}{l}\text { Translation elongation } \\
\text { dynamics }\end{array}$ & mRNA sequence & CNN classification & $\begin{array}{l}\mathrm{AUC}=0.88,0.83 \\
\text { respectively }\end{array}$ & Yeast, human \\
\hline
\end{tabular}

frames (ORFs), UTR GC content and GC content at each codon position (Neymotin et al., 2016; Cheng et al., 2017), were found to increase the predictive power of the models. Compared to single interpreted DNA motifs, motif associations could explain a much larger portion of the dynamic range of mRNA levels (84 vs. 57\%), suggesting that instead of single motifs and regions, the entire gene regulatory structure with specific combinations of regulatory elements defines gene expression levels (Figure 3A). This was also supported by observations of co-evolution among coding and non-coding regions across 14 related yeast species. With similar objectives, Agarwal and Schendure (Agarwal and Shendure, 2020) developed "Xpresso," which could explain 59 and $71 \%$ of variation in steady-state mRNA levels in human and mouse, respectively, based only on promoter sequences and explanatory features associated with mRNA stability. They showed that Xpresso more than doubles the accuracy of alternative sequence-based models and model interpretation revealed that promoter-proximal $\mathrm{CpG}$ dinucleotides strongly predict transcriptional activity.

To predict the tissue-specific transcriptional effects of genome variation, including rare or unseen mutations, Zhou and co. (Zhou et al., 2018a) developed a DNN-based framework termed "ExPecto." Using ExPecto to profile over 140 million promoter-proximal mutations, the authors characterized the regulatory mutation space for human RNAP II-transcribed genes, which enables probing of evolutionary constraints on gene expression and $a b$ initio prediction of mutation disease effects. A similar model was constructed using residual networks (ResNets), which are multilayer CNNs that utilize skip connections to jump over some layers (He et al., 2016), termed "ExpResNet" (Zhang et al., 2020). By utilizing almost $100 \mathrm{~kb}$ of sequence around each gene"s TSS, ExpResNet outperformed existing models, including ExPecto ( $\rho=0.80$ vs. 0.75 ), across four tested tissues. Interestingly, by comparing the performance achieved with different input sequence sizes, we can observe that the majority of regulatory information in humans is constrained to $\sim 10 \mathrm{~kb}$ of regulatory structure around the TSS ( $\rho=0.77,0.79$, 0.80 with 10,40 and $95 \mathrm{~kb}$, respectively), likely since this is sufficient for the majority of genes, whereas enhancers outside of this region are gene-specific and positioned highly variably.

\section{Predicting Protein Abundance From mRNA Sequence}

In multiple organisms, protein levels at steady state are primarily determined by mRNA levels, where up to $\sim 85 \%$ of the variation of 
protein expression can be attributed to mRNA transcription rather than protein translation (Schwanhäusser et al., 2011; Csárdi et al., 2015; Liu et al., 2016). Nevertheless, the spatial and temporal variations of mRNAs and the local availability of resources for protein biosynthesis strongly influence the relationship between protein levels and their transcripts (Liu et al., 2016). Thus, in many scenarios, transcript levels by themselves are not sufficient to predict protein levels and multiple other mRNA-related properties and processes affect translation and define the final gene expression levels. It was also shown that, due to translation rates per mRNA molecule being positively correlated with mRNA abundance, protein levels do not scale linearly with mRNA levels, but instead scale with the abundance of mRNA raised to the power of an "amplification exponent” (Csárdi et al., 2015). Li and co. (Li et al., 2017b) proposed that, to quantify translational control, the translation rate must be decomposed mathematically into two components: one that is dependent on mRNA abundance $\left(\mathrm{TR}_{\mathrm{mD}}\right)$, defining also the amplification exponent, and one that is not $\left(\mathrm{TR}_{\mathrm{mIND}}\right)$. In yeast, $\mathrm{TR}_{\mathrm{mD}}$ represented $\sim 20 \%$ of the variance in translation, whereas $\mathrm{TR}_{\mathrm{mIND}}$ constituted the remaining $\sim 80 \%$ of the variance in translation. The components were also preferentially determined by different mRNA sequence features: $\mathrm{TR}_{\mathrm{mIND}}$ by the length of the ORF and $\mathrm{TR}_{\mathrm{mD}}$ by a $\sim 60 \mathrm{nt}$ element spanining the initiating AUG and by CUB, implying that these components are under different evolutionary selective pressures.

Quantification of absolute protein and mRNA abundances for over 1,025 genes from the human Daoy medulloblastoma cell line showed that the combined contribution of mRNA levels and sequence features can explain $2 / 3$ of protein abundance variation at steady state (Vogel et al., 2010) (Figure 3B). Using multivariate adaptive regression splines (MARS), a nonlinear regression technique, the variation in protein abundance was primarily explained by translation elongation factors (31\%), with an impact similar to that of mRNA abundance (29\%). The strongest individual correlates of protein levels were translation and degradation-related features including mRNA sequence length, AA properties, upstream ORFs and $5^{\prime}$ UTR secondary structures. Interestingly, characteristics of the $3^{\prime}$ UTR explained a larger proportion of protein abundance variation (8\%) than characteristics of the $5^{\prime}$ UTR (1\%). A similar analysis performed with 824 genes in E. coli, which used PLS regression and over 100 mRNA sequence features, also derived a model that explained $2 / 3$ of the total variation of protein abundance (Guimaraes et al., 2014). The model suggests that protein abundance is primarily determined by the transcript level (53\%) and by effectors of translation elongation (12\%), which included both CUB and specific AA composition, whereas only a small fraction of the variation is explained by translation initiation (1\%). Lahtvee and co. (Lahtvee et al., 2017). measured absolute abundances of 5,354 mRNAs and 2,198 proteins in yeast under different environmental conditions, showing that the overall correlation between mRNA and protein abundances across all conditions is much higher for a subset of 202 differentially expressed proteins than all of them (avg. $r=0.88$ vs. 046 ). On a subset of 1,117 proteins, for which translation efficiencies were calculated, MARS detected that
mRNA abundance and translation elongation were the dominant factors controlling protein synthesis, explaining 61 and $15 \%$ of its variance, with only a small fraction (4\%) explained by translation initiation (Figure 3B).

On the other hand, multiple recent studies show that general mRNA features control a much larger fraction of the variance in translation rates or protein abundance than previously realized. For instance, $\mathrm{Li}$ and co. (Li et al., 2019a) quantified the contributions of mRNA sequence features to predicting translation rates using LR across multiple organisms, including yeast and human, where they specified 81 and $42 \%$ of the variance in translation rates, respectively. The identified informative mRNA features included similar ones as found in previous studies: 5' UTR secondary structures, nucleotides flanking AUG, upstream ORFs, ORF length and CUB (Vogel et al., 2010; Neymotin et al., 2016; Cheng et al., 2017).

Eraslan and co. (Eraslan et al., 2019b) also showed that a large fraction of protein abundance variation can be predicted from mRNA sequence in humans, by analyzing 11,575 proteins across 29 human tissues using matched transcriptomes and proteomes. Their initial LR model explained on average $22 \%$ of the variance from sequence alone, and by including additional experimentally characterized interactions and modifications, including mRNA methylation (Zhao et al., 2017), miRNA and RBP binding sites (Mayr, 2017) and post-translational modifications (Millar et al., 2019), the explained variance increased to $62 \%$. Their findings support much of the previously identified mRNA regulatory elements and also uncover new sequence motifs across the whole transcript. Importantly, they also developed a new metric of codon optimality, termed "Protein-to-mRNA adaptation index" that captures the effects of codon frequency on protein synthesis and degradation. Terai and Asai (Terai and Asai, 2020) evaluated six types of structural features in E. coli, including mRNA accessibility, which is the probability that a given region around the start codon has no base-paired nucleotides. When calculated by a log-linear model, accessibility showed the highest correlation with protein abundance. This was significantly higher than the widely used minimum free energy ( $\rho=0.71$ vs. 0.55 ), and combining it with activity of the Shine-Dalgarno sequence yielded a highly accurate method for predicting protein abundance $(\rho=0.76)$. Moreover, similarly as in eukaryotes, secondary structures in bacterial mRNAs were shown to be highly important for protein production and to generally limit translation initiation in a large-scale assay involving 244,000 designed sequences with varying features (Cambray et al., 2018).

Deep learning was recently applied to prediction of translation initiation sites in a method termed "TITER" (Zhang et al., 2017b), using HTS data quantitatively profiling initiating ribosomes (QTI-seq) at single-nucleotide resolution (Gao et al., 2015). Using a hybrid CNN + RNN approach, TITER integrates the prior preference of TIS codon composition with translation initiation features extracted from the surrounding sequence to greatly outperform other state-of-the-art methods in predicting the initiation sites. The method captures the sequence motifs of different start codons, including a Kozak sequence-like motif for AUG, and quantifies mutational effects on translation initiation. 
TABLE 4 | The current advantages, disadvantages and further challenges of machine learning methods in genetics and genomics.

\section{Deep methods}

Shallow methods

Advantages Scale effectively with data and support use of latest computational and technological advances, including large genomic datasets and results of HTS technologies (Barshai et al., 2020) Ability to automatically learn features from raw input data and unlock an additional level of information from it (Barshai et al., 2020; Zrimec et al., 2020)

Ability to learn and approximate complex functions without prior assumptions, frequently achieving improved predictive power (Barshai et al., 2020)

Capability to integrate multiple pre-processing steps into a single end-toend model (Eraslan et al., 2019a)

Ability to effectively model multimodal data (Eraslan et al., 2019a) Highly useful as experiment simulators due to the ability to generalize over an experimental dataset (Barshai et al., 2020)

Easily adaptable to different domains and applications, with transfer learning on pre-trained deep networks accelerating training and improving performance

Disadvantages Dependence on accurately labeled data: cannot achieve higher accuracy than that allowed by the noise inherent to the given experimental target labels (Li et al., 2019b; Barshai et al., 2020)

Data driven instead of hypothesis driven modeling (Barshai et al., 2020)
Dependence on large amounts of data (at least thousands of training examples) and specialized computational resources (e.g. GPUs)

Potential problems with generalizability, as can be overfit to the experiment rather than biological function (Barshai et al., 2020)

Potential lack of model interpretability (Zou et al., 2019; Barshai et al., 2020)

Dependence on feature engineering

Many different algorithms each with its own advantages and disadvantages can be daunting and require extensive specialized study (Hastie et al., 2013) Cannot unlock information directly from nucleotide sequence (Azodi et al., 2020; Zrimec et al., 2020)

Challenges

Methods to interpret heterogeneous multi-omic and highly dimensional data (Azodi et al., 2020)

Methods and high quality datasets to benchmark existing and new interpretation strategies (Azodi et al., 2020)

Methods to join findings from multiple interpretation strategies into more complete and coherent interpretations of both models (Azodi et al., 2020) and the studied molecular phenomena

Making interpretable ML more accessible to biologists by further lowering the entry barriers and requirements of computational knowledge (Azodi et al., 2020)

Another DNN framework, termed "ROSE" was used to analyze translation elongation dynamics in both human and yeast via ribosome stalling, which is manifested by the local accumulation of ribosomes at specific codon positions of mRNA (Zhang et al., 2017c). ROSE estimates the probability of a ribosome stalling event occurring at each genomic location, achieving higher prediction accuracy than conventional prediction models such as gkm-SVM with AUC increases by up to $18.4 \%$.

\section{DISCUSSION}

As can be surmised from the presented ML results (Table1, Table 2, and Table 3), deep methods frequently outperform shallow ones, and we outline the main advantages, disadvantages and challenges of these approaches in Table 4 . The capability of DNNs to more accurately recapitulate experimental data stems mainly from their ability to extract information directly from the raw input nucleotide sequences, automatically learning regulatory grammar (Figures 2B,D), which boosts predictive accuracy (Zrimec et al., 2020). However, although multiple different methods exist for interpreting deep methods, many are a work in progress and no explicit solutions currently exist to benchmark these methods or to combine the findings into more complete and coherent interpretations (Azodi et al., 2020). Nevertheless, ML in general lowers the entry barrier to development of new models and saves research time by abstracting mathematical details (Eraslan et al., 2019a), though this has also been used to criticize such approaches, as they rely on data driven instead of hypothesis driven modeling (Barshai et al. 2020). An important limitation of all ML methods is their dependence on accurately labeled data, since they cannot achieve higher accuracy than that allowed by the noise inherent to the given target labels (Li et al., 2019b; Barshai et al., 2020). For instance, in vivo measurements, such as those produced by ChIP-seq, ATAC-seq and DNAse-seq, are prone to experimental noise and technological artifacts and subject to the complexity of the cellular environment, affected by chromatin structure and nucleosome positioning, thus concealing the full picture of DBP-DNA interactions. Alternatively, in vitro methods, such as PBMs, HT-SELEX and BunDLE-seq, can capture purely direct protein-nucleic acid interactions or cooperative binding of specific factors and allow sampling of the full spectrum of binding sites (Barshai et al., 2020). Fortunately, novel computational methods allow researchers to easily estimate the noise-constrained upper bound of ML regression model performance (Li et al., 2019b). 
Despite the knowledge that whole regulatory structures are involved in gene expression, the majority of approaches still focus only on single regulatory or coding regions. For instance, with mRNA abundance prediction, the contribution of the separate parts of the gene regulatory structure has been quantified only in yeast (Zrimec et al., 2020) (Figure 3A). The results across the remaining studies are highly variable, likely due to using very different methods and protocols (Table 2 and Table 3). The trend of using whole regulatory structures is however more common with protein abundance prediction, where, apart from mRNA abundance, also the parts involved in translational processing have been quantified across all three major model organisms (Figure 3B). Nevertheless, both the fact that these studies were performed using classical shallow models as well as results from other studies suggest that there is potential for improvement. For instance, results from multiple studies focusing on individual regions show that a much higher amount of information can be extracted from these regions [Table 2: e.g. $62 \%$ of protein abundance variation explained from yeast $5^{\prime}$ UTRs with DNNs (Decoene et al., 2018)] than was achieved with shallow learning on whole mRNAs in Figure 3B. Based on other results, we can also presume that it is possible to not only further boost predictive performance but also uncover new mRNA regulatory grammar.

Pooling the highest-scoring results across organisms in an information-centric view of the central dogma of molecular biology (Figure 3C) suggests that about $2 / 3$ of the variation of mRNA and protein levels can be explained from DNA sequence. Unequal approaches were employed however, with deep learning used only with mRNA abundance modeling. Here, the lower results with $H$. sapiens might be a result of accounting for only promoter regions and mRNA stability-associated features in the model (Agarwal and Shendure, 2020), though our own analysis had shown that these stability features alone can explain $38 \%$ of the mRNA abundance variation in yeast (Zrimec et al., 2020). Interestingly, by omitting the mRNA abundance component from protein abundance predictions, we can observe the possibility of an increasing trend of explained variance with increasing organism complexity (Figure 3C: $13-40 \%$ from bacteria to human). This would indicate that mRNAs of multicellular eukaryotes carry more regulatory information involved in translation than those of unicellular eukaryotes and prokaryotes. It might also reflect the fact that gene expression regulation is more intricate in multicellular organisms due to the multiple additional regulatory processes that control expression of a much more complex set of biomolecules and phenotypes than in unicellular organisms (Benelli et al., 2016).

Regulatory information seems to be localized around the gene, as multiple studies show that the region spanning $<10 \mathrm{~kb}$ around the TSS has the largest measurable effect on gene expression, likely as the majority of regulatory signals are clustered in this region in most genes and organisms (Agarwal and Shendure, 2020; Ansariola et al., 2020; Zrimec et al., 2020). Enhancers on the other hand are highly variably spaced and act in a gene-specific manner, which makes them much harder to recognize, and also requires processing enormous sizes of input sequences (e.g. $>100 \mathrm{~kb}$ upstream of genes in human data) that require more training resources. Therefore, the true effect of such regions is still hard to decipher. Procedures handling larger input sequence sizes or whole genomes will likely lead to improved analysis and quantification of the contributions of enhancers to gene expression control, in relation to other parts of the regulatory structure (Singh et al., 2019; Tang et al., 2020). Another potential trend is building DNNs using biophysical (Tareen and Kinney, 2019) or physicochemical properties (Yang et al., 2017; Liu et al., 2020), as deep models trained on these features might uncover novel patterns in data and lead to improved understanding of the physicochemical principles of protein-nucleic acid regulatory interactions, as well as aid model interpretability. Other novel approaches include: 1) modifying DNN properties to improve recovery of biologically meaningful motif representations (Koo and Ploenzke, 2021), 2) transformer networks (Devlin et al., 2018) and attention mechanisms (Vaswani et al., 2017), widely used in protein sequence modeling (Jurtz et al., 2017; Rao et al., 2019; Vig et al., 2020; Repecka et al., 2021), 3) graph convolutional neural networks, a class of DNNs that can work directly on graphs and take advantage of their structural information, with the potential to give us great insights if we can reframe genomics problems as graphs (Cranmer et al., 2020; Strokach et al., 2020), and 4) generative modeling (Foster, 2019), which may help exploit current knowledge in designing synthetic sequences with desired properties (Killoran et al., 2017; Wang Y. et al., 2020). With the latter, unsupervized training is used with approaches including: 1) autoencoders, which learn efficient representations of the training data, typically for dimensionality reduction (Way and Greene, 2018) or feature selection (Xie et al., 2017), 2) generative adversarial networks, which learn to generate new data with the same statistics as the training set (Wang Y. et al., 2020; Repecka et al., 2021), and 3) deep belief networks, which learn to probabilistically reconstruct their inputs, acting as feature detectors, and can be further trained with supervision to build efficient classifiers (Bu et al., 2017). Moreover, the advent of single-cell HTS technologies such as single-cell RNA-seq will offer many novel research opportunities, including modeling of cell-type or cell-state specific enhancer or TFBS activations and chromatin changes (Angermueller et al., 2017; Gustafsson et al., 2020; Kawaguchi et al., 2021).

To conclude, the application of ML in genomics has augmented experimental methods and facilitated accumulating a vast amount of knowledge on gene expression regulation. DNNs, due to their ability to learn biologically relevant information directly from sequence, while performing similarly to or better than classical approaches, are the method of choice for quantifying gene expression and interpreting the predictive features hidden in nucleotide sequence data. DNN-isolated features can be as predictive as models relying on experimental ChIP-seq data (Agarwal and Shendure, 2020), suggesting that current computational approaches are achieving a level of accuracy that might soon allow substituting wet-lab HTS experiments with fully 
computational pipelines (Keilwagen et al., 2019). Such pipelines can also become indispensable for analysis of human disease-associated regulatory mutations, identifying clinically relevant noncoding variants and expression perturbations, grouping patients in drug treatment trials, disease subtyping as well as personalized treatment (Zhou et al., 2018a; Dagogo-Jack and Shaw, 2018). Since controlling the expression of genes is also one of the key challenges of synthetic biology, the computational models represent excellent starting points in procedures to predictably design regulatory sequences, control protein expression and fine-tune biosynthetic pathways in both prokaryotic and eukaryotic systems (Nielsen and Keasling, 2016; de Jongh et al., 2020; Wang H. et al., 2020).

For readers willing to learn and apply some of the discussed ML approaches, many excellent resources exist, including: 1) specialized packages for model development and interpretation, such as "DragoNN" (https://kundajelab.github.io/dragonn/) (Movva et al., 2019) "Janggu" (https://github.com/ BIMSBbioinfo/janggu) (Kopp et al., 2020) and "Pysster" (https:/github.com/budach/pysster) (Budach and Marsico, 2018), 2) repositories of trained models, such as "Kipoi" (https://kipoi.org/) (Avsec et al., 2019), 3) other genomics tutorials and code examples (https:/github.com/vanessajurtz/ lasagne4bio) (Jurtz et al., 2017), as well as 4) resources with a much broader scope than mere genomics, including online

\section{REFERENCES}

Abe, N., Dror, I., Yang, L., Slattery, M., Zhou, T., Bussemaker, H. J., et al. (2015). Deconvolving the Recognition of DNA Shape from Sequence. Cell 161, 307-318. doi:10.1016/j.cell.2015.02.008

Agarwal, V., and Shendure, J. (2020). Predicting mRNA Abundance Directly from Genomic Sequence Using Deep Convolutional Neural Networks. Cell Rep 31, 107663. doi:10.1016/j.celrep.2020.107663

Alipanahi, B., Delong, A., Weirauch, M. T., and Frey, B. J. (2015). Predicting the Sequence Specificities of DNA- and RNA-Binding Proteins by Deep Learning. Nat. Biotechnol. 33, 831-838. doi:10.1038/nbt.3300

Ancona, M., Ceolini, E., Öztireli, C., and Gross, M. (2017). Towards Better Understanding of Gradient-Based Attribution Methods for Deep Neural Networks. Ithaca, NY: arXiv [cs.LG].

Angermueller, C., Lee, H. J., Reik, W., and Stegle, O. (2017). DeepCpG: Accurate Prediction of Single-Cell DNA Methylation States Using Deep Learning. Genome Biol. 18, 67. doi:10.1186/s13059-017-1189-z

Angermueller, C., Pärnamaa, T., Parts, L., and Stegle, O. (2016). Deep Learning for Computational Biology. Mol. Syst. Biol. 12, 878. doi:10.15252/msb.20156651

Angov, E., Hillier, C. J., Kincaid, R. L., and Lyon, J. A. (2008). Heterologous Protein Expression Is Enhanced by Harmonizing the Codon Usage Frequencies of the Target Gene with Those of the Expression Host. PLoS One 3, e2189. doi:10. 1371/journal.pone.0002189

Angus, G., and Eyuboglu, S. (2018). "Regulatory Activity Prediction with Attention-Based Models," in 32nd Conference on Neural Information Processing Systems (. NIPS 2018).

Anna, A., and Monika, G. (2018). Splicing Mutations in Human Genetic Disorders: Examples, Detection, and Confirmation. J. Appl. Genet. 59, 253-268. doi:10. 1007/s13353-018-0444-7

Ansariola, M., Fraser, Valerie. N., Filichkin, Sergei. A., Ivanchenko, Maria. G., Bright, Zachary. A., Gould, Russell. A., et al. (2020). Accurate Transcription Start Sites Enable Mining for the Cis-Regulatory Determinants of Tissue Specific Gene Expression. Cold Spring Harbor, NY: Cold Spring Harbor Laboratory. doi:10. $1101 / 2020.09 .01 .278424$ courses (https://www.coursera.org/specializations/deeplearning) and books (https://github.com/ageron/handson-ml2) (Géron, 2019).

\section{AUTHOR CONTRIBUTIONS}

$\mathrm{JZ}, \mathrm{FB}$, and AZ conceptualized the project; JZ performed the literature research and wrote the paper; $\mathrm{FB}$ contributed to the literature research on translation; $\mathrm{MK}$ contributed to the introduction and discussion; VG contributed to the literature research and text on codon usage; FB, AZ and VG contributed to writing the final manuscript.

\section{FUNDING}

The study was supported by SciLifeLab funding and Swedish Research council (Vetenskapsrådet) starting Grant No. 201905356.

\section{ACKNOWLEDGMENTS}

We thank Sandra Viknander for insightful discussions on deep neural networks and their applications.

Arbiza, L., Gronau, I., Aksoy, B. A., Hubisz, M. J., Gulko, B., Keinan, A., et al. (2013). Genome-wide Inference of Natural Selection on Human Transcription Factor Binding Sites. Nat. Genet. 45, 723-729. doi:10.1038/ ng. 2658

Avsec, Ž., Weilert, M., Shrikumar, A., Krueger, S., Alexandari, A., Dalal, K., et al. (2021). Base-resolution Models of Transcription-Factor Binding Reveal Soft Motif Syntax. Nat. Genet. 53, 354-366. doi:10.1038/s41588-021-00782-6

Avsec, Ž., Kreuzhuber, R., Israeli, J., Xu, N., Cheng, J., Shrikumar, A., et al. (2019). The Kipoi Repository Accelerates Community Exchange and Reuse of Predictive Models for Genomics. Nat. Biotechnol. 37, 592-600. doi:10.1038/ s41587-019-0140-0

Azodi, C. B., Tang, J., and Shiu, S.-H. (2020). Opening the Black Box: Interpretable Machine Learning for Geneticists. Trends Genet. 36, 442-455. doi:10.1016/j.tig. 2020.03.005

Bansal, M., Kumar, A., and Yella, V. R. (2014). Role of DNA Sequence Based Structural Features of Promoters in Transcription Initiation and Gene Expression. Curr. Opin. Struct. Biol. 25, 77-85. doi:10.1016/j.sbi.2014.01.007

Barrett, L. W., Fletcher, S., and Wilton, S. D. (2012). Regulation of Eukaryotic Gene Expression by the Untranslated Gene Regions and Other Non-coding Elements. Cell. Mol. Life Sci. 69, 3613-3634. doi:10.1007/s00018-012-0990-9

Barshai, M., Tripto, E., and Orenstein, Y. (2020). Identifying Regulatory Elements via Deep Learning. Annu. Rev. Biomed. Data Sci. 3, 315-338. doi:10.1146/ annurev-biodatasci-022020-021940

Bello, I., Zoph, B., Vaswani, A., Shlens, J., and Le, Q. V. (2019). Attention Augmented Convolutional Networks. Ithaca, NY: arXiv [cs.CV].

Benelli, D., La Teana, A., and Londei, P. (2016). "Evolution of Translational Initiation: From Archaea to Eukarya," in Evolution of the Protein Synthesis Machinery and its Regulation. (Berlin, Germany: Springer), 61-79.

Benveniste, D., Sonntag, H.-J., Sanguinetti, G., and Sproul, D. (2014). Transcription Factor Binding Predicts Histone Modifications in Human Cell Lines. Proc. Natl. Acad. Sci. U. S. A. 111, 13367-13372. doi:10.1073/pnas.1412081111

Berger, M. F., Philippakis, A. A., Qureshi, A. M., He, F. S., Estep, P. W., and Bulyk, M. L. (2006). Compact, Universal DNA Microarrays to Comprehensively Determine Transcription-Factor Binding Site Specificities. Nat. Biotechnol. 24, 1429-1435. doi:10.1038/nbt1246 
Bergstra, J., Komer, B., Eliasmith, C., Yamins, D., and Cox, D. D. (2015). Hyperopt: a Python Library for Model Selection and Hyperparameter Optimization. Comput. Sci. Discov. 8, 014008. doi:10.1088/1749-4699/8/1/014008

Bishop, E. P., Rohs, R., Parker, S. C. J., West, S. M., Liu, P., Mann, R. S., et al. (2011). A Map of Minor Groove Shape and Electrostatic Potential from Hydroxyl Radical Cleavage Patterns of DNA. ACS Chem. Biol. 6, 1314-1320. doi:10.1021/ cb200155t

Blackwell, T. K., and Weintraub, H. (1990). Differences and Similarities in DNABinding Preferences of MyoD and E2A Protein Complexes Revealed by Binding Site Selection. Science 250, 1104-1110. doi:10.1126/science.2174572

Bogard, N., Linder, J., Rosenberg, A. B., and Seelig, G. (2019). A Deep Neural Network for Predicting and Engineering Alternative Polyadenylation. Cell 178, 91-106. doi:10.1016/j.cell.2019.04.046

Bonde, M. T., Pedersen, M., Klausen, M. S., Jensen, S. I., Wulff, T., Harrison, S., et al. (2016). Predictable Tuning of Protein Expression in Bacteria. Nat. Methods 13, 233-236. doi:10.1038/nmeth.3727

Browning, D. F., and Busby, S. J. (2004). The Regulation of Bacterial Transcription Initiation. Nat. Rev. Microbiol. 2, 57-65. doi:10.1038/nrmicro787

Brukner, I., Sanchez, R., Suck, D., and Pongor, S. (1995). Sequence-dependent Bending Propensity of DNA as Revealed by DNase I: Parameters for Trinucleotides. EMBO J. 14, 1812-1818. doi:10.1002/j.1460-2075.1995. tb07169.x

Bu, H., Gan, Y., Wang, Y., Zhou, S., and Guan, J. (2017). A New Method for Enhancer Prediction Based on Deep Belief Network. BMC Bioinformatics 18, 418. doi:10.1186/s12859-017-1828-0

Budach, S., and Marsico, A. (2018). Pysster: Classification of Biological Sequences by Learning Sequence and Structure Motifs with Convolutional Neural Networks. Bioinformatics 34, 3035-3037. doi:10.1093/bioinformatics/ bty 222

Buenrostro, J. D., Giresi, P. G., Zaba, L. C., Chang, H. Y., and Greenleaf, W. J. (2013). Transposition of Native Chromatin for Fast and Sensitive Epigenomic Profiling of Open Chromatin, DNA-Binding Proteins and Nucleosome Position. Nat. Methods 10, 1213-1218. doi:10.1038/nmeth.2688

Cambray, G., Guimaraes, J. C., and Arkin, A. P. (2018). Evaluation of 244,000 Synthetic Sequences Reveals Design Principles to Optimize Translation in Escherichia coli. Nat. Biotechnol. 36, 1005-1015. doi:10.1038/nbt.4238

Cambray, G., Guimaraes, J. C., Mutalik, V. K., Lam, C., Mai, Q.-A., Thimmaiah, T., et al. (2013). Measurement and Modeling of Intrinsic Transcription Terminators. Nucleic Acids Res. 41, 5139-5148. doi:10.1093/nar/gkt163

Carbone, A., Zinovyev, A., and Képès, F. (2003). Codon Adaptation index as a Measure of Dominating Codon Bias. Bioinformatics 19, 2005-2015. doi:10. 1093/bioinformatics/btg272

Castillo-Davis, C. I., Hartl, D. L., and Achaz, G. (2004). cis-Regulatory and Protein Evolution in Orthologous and Duplicate Genes. Genome Res. 14, 1530-1536. doi:10.1101/gr.2662504

Chen, C., Hou, J., Shi, X., Yang, H., Birchler, J. A., and Cheng, J. (2021). DeepGRN: Prediction of Transcription Factor Binding Site across Cell-Types Using Attention-Based Deep Neural Networks. BMC Bioinformatics 22, 38. doi:10. 1186/s12859-020-03952-1

Chen, J., Darst, S. A., and Thirumalai, D. (2010). Promoter Melting Triggered by Bacterial RNA Polymerase Occurs in Three Steps. Proc. Natl. Acad. Sci. U. S. A. 107, 12523-12528. doi:10.1073/pnas.1003533107

Chen, L., and Capra, J. A. (2020). Learning and Interpreting the Gene Regulatory Grammar in a Deep Learning Framework. Plos Comput. Biol. 16, e1008334. doi:10.1371/journal.pcbi.1008334

Chen, W., Zhang, X., Brooker, J., Lin, H., Zhang, L., and Chou, K. C. (2015). PseKNC-General: a Cross-Platform Package for Generating Various Modes of Pseudo Nucleotide Compositions. Bioinformatics 31, 119-120. doi:10.1093/ bioinformatics/btu602

Chen, Y., Pai, A. A., Herudek, J., Lubas, M., Meola, N., Järvelin, A. I., et al. (2016). Principles for RNA Metabolism and Alternative Transcription Initiation within Closely Spaced Promoters. Nat. Genet. 48, 984-994. doi:10.1038/ng.3616

Cheng, J., Maier, K. C., Avsec, Ž., Rus, P., and Gagneur, J. (2017). Cis-regulatory Elements Explain Most of the mRNA Stability Variation across Genes in Yeast. RNA 23, 1648-1659. doi:10.1261/rna.062224.117

Chiu, T.-P., Xin, B., Markarian, N., Wang, Y., and Rohs, R. (2020). TFBSshape: an Expanded Motif Database for DNA Shape Features of Transcription Factor Binding Sites. Nucleic Acids Res. 48, D246-D255. doi:10.1093/nar/gkz970
Clément, Y., Torbey, P., and Gilardi-Hebenstreit, P. (2018). Genome-wide Enhancer-Gene Regulatory Maps in Two Vertebrate Genomes. Cold Spring Harbor, NY: bioRxiv.

Cohn, D., Zuk, O., and Kaplan, T. (2018). Enhancer Identification Using Transfer and Adversarial Deep Learning of DNA Sequences. Cold Spring Harbor Lab. 264200. doi:10.1101/264200

Cranmer, M., Sanchez-Gonzalez, Alvaro., Battaglia, Peter., Xu, Rui., Cranmer, Kyle., Spergel, David., et al. (2020). Discovering Symbolic Models from Deep Learning with Inductive Biases. Ithaca, NY: arXiv [cs.LG].

Csárdi, G., Franks, A., Choi, D. S., Airoldi, E. M., and Drummond, D. A. (2015). Accounting for Experimental Noise Reveals that mRNA Levels, Amplified by post-transcriptional Processes, Largely Determine Steady-State Protein Levels in Yeast. Plos Genet. 11, e1005206. doi:10.1371/journal.pgen.1005206

Cuperus, J. T., Groves, B., and Kuchina, A. (2017). Deep Learning of the Regulatory Grammar of Yeast 5' Untranslated Regions from 500,000 Random Sequences. Genome Res. 27, 1-10. doi:10.1101/gr.224964.117

Curran, K. A., Crook, N. C., Karim, A. S., Gupta, A., Wagman, A. M., and Alper, H. S. (2014). Design of Synthetic Yeast Promoters via Tuning of Nucleosome Architecture. Nat. Commun. 5, 4002. doi:10.1038/ncomms5002

Curran, K. A., Morse, N. J., Markham, K. A., Wagman, A. M., Gupta, A., and Alper, H. S. (2015). Short Synthetic Terminators for Improved Heterologous Gene Expression in Yeast. ACS Synth. Biol. 4, 824-832. doi:10.1021/sb5003357

Dagogo-Jack, I., and Shaw, A. T. (2018). Tumour Heterogeneity and Resistance to Cancer Therapies. Nat. Rev. Clin. Oncol. 15, 81-94. doi:10.1038/nrclinonc. 2017.166

de Boer, C. G., Vaishnav, E. D., Sadeh, R., Abeyta, E. L., Friedman, N., and Regev, A. (2020). Deciphering Eukaryotic Gene-Regulatory Logic with 100 Million Random Promoters. Nat. Biotechnol. 38, 56-65. doi:10.1038/s41587-0190315-8

de Jongh, R. P. H., van Dijk, A. D. J., Julsing, M. K., Schaap, P. J., and de Ridder, D. (2020). Designing Eukaryotic Gene Expression Regulation Using Machine Learning. Trends Biotechnol. 38, 191-201. doi:10.1016/j.tibtech.2019.07.007

Decoene, T., Peters, G., De Maeseneire, S. L., and De Mey, M. (2018). Toward Predictable 5'UTRs in Saccharomyces cerevisiae: Development of a yUTR Calculator. ACS Synth. Biol. 7, 622-634. doi:10.1021/acssynbio.7b00366

Devlin, J., Chang, M.-W., Lee, K., and Toutanova, K. (2018). BERT: Pre-training of Deep Bidirectional Transformers for Language Understanding. Ithaca, NY: arXiv [cs.CL].

Dhillon, N., Shelansky, R., Townshend, B., Jain, M., Boeger, H., Endy, D., et al. (2020). Permutational Analysis of Saccharomyces cerevisiae Regulatory Elements. Synth. Biol. 5, ysaa007. doi:10.1093/synbio/ysaa007

Dillon, S. C., and Dorman, C. J. (2010). Bacterial Nucleoid-Associated Proteins, Nucleoid Structure and Gene Expression. Nat. Rev. Microbiol. 8, 185-195. doi:10.1038/nrmicro2261

Ding, W., Cheng, J., Guo, D., Mao, L., Li, J., Lu, L., et al. (2018). Engineering the 5' UTR-Mediated Regulation of Protein Abundance in Yeast Using Nucleotide Sequence Activity Relationships. ACS Synth. Biol. 7, 2709-2714. doi:10.1021/ acssynbio. 8 b00127

Dvir, S., Velten, L., Sharon, E., and Zeevi, D. (2013). Deciphering the Rules by Which 5'-UTR Sequences Affect Protein Expression in Yeast. Proc. Natl. Acad. Sci. 110, E2792-E2801. doi:10.1073/pnas.1222534110

Einav, T., and Phillips, R. (2019). How the Avidity of Polymerase Binding to the -35/-10 Promoter Sites Affects Gene Expression. Proc. Natl. Acad. Sci. U. S. A. 116, 13340-13345. doi:10.1073/pnas.1905615116

ENCODE Project Consortium (2012). An Integrated Encyclopedia of DNA Elements in the Human Genome. Nature 489, 57-74. doi:10.1038/ nature 11247

Eraslan, B., Wang, D., Gusic, M., Prokisch, H., Hallström, B. M., Uhlén, M., et al. (2019). Quantification and Discovery of Sequence Determinants of Proteinper-mRNA Amount in 29 Human Tissues. Mol. Syst. Biol. 15. doi:10.15252/ msb. 20188513

Eraslan, G., Avsec, Ž., Gagneur, J., and Theis, F. J. (2019). Deep Learning: New Computational Modelling Techniques for Genomics. Nat. Rev. Genet. 20, 389-403. doi:10.1038/s41576-019-0122-6

Espah Borujeni, A., Cetnar, D., Farasat, I., Smith, A., Lundgren, N., and Salis, H. M. (2017). Precise Quantification of Translation Inhibition by mRNA Structures that Overlap with the Ribosomal Footprint in N-Terminal Coding Sequences. Nucleic Acids Res. 45, 5437-5448. doi:10.1093/nar/gkx061 
Espinar, L., Schikora Tamarit, M. À., Domingo, J., and Carey, L. B. (2018). Promoter Architecture Determines Cotranslational Regulation of mRNA. Genome Res. 28, 509-518. doi:10.1101/gr.230458.117

Roadmap Epigenomics Consortium (2015). Integrative Analysis of 111 Reference Human Epigenomes. Nature 518, 317-330. doi:10.1038/nature14248

Feklístov, A., Sharon, B. D., Darst, S. A., and Gross, C. A. (2014). Bacterial Sigma Factors: a Historical, Structural, and Genomic Perspective. Annu. Rev. Microbiol. 68, 357-376. doi:10.1146/annurev-micro-092412-155737

Ferreira, M., Ventorim, R., Almeida, E., Silveira, S., and Silveira, W. (2020). Protein Abundance Prediction through Machine Learning Methods. Cold Spring Harbor, NY: Cold Spring Harbor Laboratory. doi:10.1101/2020.09. 17.302182

Fletez-Brant, C., Lee, D., McCallion, A. S., and Beerkmer, M. A. S. V. M. (2013). A Web Server for Identifying Predictive Regulatory Sequence Features in Genomic Data Sets. Nucleic Acids Res. 41, W544-W556. doi:10.1093/nar/ gkt519

Foster, D. (2019). Generative Deep Learning: Teaching Machines to Paint, Write, Compose, and Play. Sebastopol, CA: 'O’Reilly Media, Inc.'.

Fu, H., Liang, Y., Zhong, X., Pan, Z., Huang, L., Zhang, H., et al. (2020). Codon Optimization with Deep Learning to Enhance Protein Expression. Sci. Rep. 10, 17617. doi:10.1038/s41598-020-74091-z

Fujimoto, M. S., Bodily, Paul. M., Lyman, Cole. A., Jacobsen, Andrew. J., Snell, Quinn., and Clement, Mark. J. (2017). "Modeling Global and Local Codon Bias with Deep Language Models," in 2017 IEEE 17th International Conference on Bioinformatics and Bioengineering (BIBE), 151-156.

Gao, X., Wan, J., Liu, B., Ma, M., Shen, B., and Qian, S.-B. (2015). Quantitative Profiling of Initiating Ribosomes In Vivo. Nat. Methods 12, 147-153. doi:10. 1038/nmeth.3208

Gaspar, P., Oliveira, J. L., Frommlet, J., Santos, M. A. S., and Moura, G. (2012). EuGene: Maximizing Synthetic Gene Design for Heterologous Expression. Bioinformatics 28, 2683-2684. doi:10.1093/bioinformatics/bts465

Geggier, S., and Vologodskii, A. (2010). Sequence Dependence of DNA Bending Rigidity. Proc. Natl. Acad. Sci. U. S. A. 107, 15421-15426. doi:10.1073/pnas. 1004809107

Géron, A. (2019). Hands-On Machine Learning with Scikit-Learn, Keras, and TensorFlow: Concepts, Tools, and Techniques to Build Intelligent Systems. Sebastopol, CA: 'O’Reilly Media, Inc.'.

Ghaemmaghami, S., Huh, W.-K., Bower, K., Howson, R. W., Belle, A., Dephoure, N., et al. (2003). Global Analysis of Protein Expression in Yeast. Nature 425, 737-741. doi:10.1038/nature02046

Ghandi, M., Lee, D., Mohammad-Noori, M., and Beer, M. A. (2014). Enhanced Regulatory Sequence Prediction Using Gapped K-Mer Features. Plos Comput. Biol. 10, e1003711. doi:10.1371/journal.pcbi.1003711

Gibney, E. R., and Nolan, C. M. (2010). Epigenetics and Gene Expression. Heredity 105, 4-13. doi:10.1038/hdy.2010.54

Gould, N., Hendy, O., and Papamichail, D. (2014). Computational Tools and Algorithms for Designing Customized Synthetic Genes. Front. Bioeng. Biotechnol. 2, 41. doi:10.3389/fbioe.2014.00041

Grant, C. E., Bailey, T. L., and Noble, W. S. (2011). FIMO: Scanning for Occurrences of a Given Motif. Bioinformatics 27, 1017-1018. doi:10.1093/ bioinformatics/btr064

Grant, C. E., Johnson, J., Bailey, T. L., and Noble, W. S. (2016). MCAST: Scanning for Cis-Regulatory Motif Clusters. Bioinformatics 32, 1217-1219. doi:10.1093/ bioinformatics/btv750

Grossman, S. R., Zhang, X., Wang, L., Engreitz, J., Melnikov, A., Rogov, P., et al. (2017). Systematic Dissection of Genomic Features Determining Transcription Factor Binding and Enhancer Function. Proc. Natl. Acad. Sci. U. S. A. 114, E1291-E1300. doi:10.1073/pnas.1621150114

Guimaraes, J. C., Rocha, M., and Arkin, A. P. (2014). Transcript Level and Sequence Determinants of Protein Abundance and Noise in Escherichia coli. Nucleic Acids Res. 42, 4791-4799. doi:10.1093/nar/gku126

Guo, Z., and Sherman, F. (1996). 3'-end-forming Signals of Yeast mRNA. Trends Biochem. Sci. 21, 477-481. doi:10.1016/s0968-0004(96)10057-8

Gupta, A., and Rush, A. M. (2017). Dilated Convolutions for Modeling LongDistance Genomic Dependencies. Ithaca, NY: arXiv [q-bio.GN].

Gustafsson, J., Held, F., Robinson, J. L., Björnson, E., Jörnsten, R., and Nielsen, J. (2020). Sources of Variation in Cell-type RNA-Seq Profiles. PLoS One 15, e0239495. doi:10.1371/journal.pone.0239495
Haberle, V., and Stark, A. (2018). Eukaryotic Core Promoters and the Functional Basis of Transcription Initiation. Nat. Rev. Mol. Cel Biol. 19, 621-637. doi:10. 1038/s41580-018-0028-8

Hahn, M. W. (2007). Detecting Natural Selection on Cis-Regulatory DNA. Genetica 129, 7-18. doi:10.1007/s10709-006-0029-y

Hammar, P., Leroy, P., Mahmutovic, A., Marklund, E. G., Berg, O. G., and Elf, J. (2012). The Lac Repressor Displays Facilitated Diffusion in Living Cells. Science 336, 1595-1598. doi:10.1126/science.1221648

Hanson, G., and Coller, J. (2018). Codon Optimality, Bias and Usage in Translation and mRNA Decay. Nat. Rev. Mol. Cel Biol. 19, 20-30. doi:10.1038/nrm.2017.91

Hastie, T., Tibshirani, R., and Friedman, J. (2013). The Elements of Statistical Learning: Data Mining, Inference, and Prediction. Berlin, Germany: Springer Science \& Business Media.

He, K., Zhang, X., Ren, S., and Sun, J. (2016). “Deep Residual Learning for Image Recognition," in Proceedings of the IEEE conference on computer vision and pattern recognition, 770-778.

He, Q., Johnston, J., and Zeitlinger, J. (2015). ChIP-nexus Enables Improved Detection of In Vivo Transcription Factor Binding Footprints. Nat. Biotechnol. 33, 395-401. doi:10.1038/nbt.3121

He, W., Jia, C., Duan, Y., and Zou, Q. 70Pro. Pred. (2018). A Predictor for Discovering Sigma70 Promoters Based on Combining Multiple Features. BMC Syst. Biol. 12, 44. doi:10.1186/s12918-018-0570-1

He, Y., Shen, Z., Zhang, Q., Wang, S., and Huang, D.-S. (2020). A Survey on Deep Learning in DNA/RNA Motif Mining. Brief. Bioinform., 1-10. doi:10.1093/bib/ bbaa229

Hershberg, R., and Petrov, D. A. (2009). General Rules for Optimal Codon Choice. Plos Genet. 5, e1000556. doi:10.1371/journal.pgen.1000556

Hershberg, R., and Petrov, D. A. (2008). Selection on Codon Bias. Annu. Rev. Genet. 42, 287-299. doi:10.1146/annurev.genet.42.110807.091442

Hinnebusch, A. G., Ivanov, I. P., and Sonenberg, N. (2016). Translational Control by 5'-untranslated Regions of Eukaryotic mRNAs. Science 352, 1413-1416. doi:10.1126/science.aad9868

Hossain, A., Lopez, E., Halper, S. M., Cetnar, D. P., Reis, A. C., Strickland, D., et al. (2020). Automated Design of Thousands of Nonrepetitive Parts for Engineering Stable Genetic Systems. Nat. Biotechnol. 38, 1466-1475. doi:10.1038/s41587020-0584-2

Inukai, S., Kock, K. H., and Bulyk, M. L. (2017). Transcription Factor-DNA Binding: beyond Binding Site Motifs. Curr. Opin. Genet. Dev. 43, 110-119. doi:10.1016/j.gde.2017.02.007

Jaganathan, K., Panagiotopoulou, S. K., McRae, J. F., Darbandi, S. F., Knowles, D., Li, Y. I., et al. (2019). Predicting Splicing from Primary Sequence with Deep Learning. Cell 176 (3), 535-548. doi:10.1016/j.cell.2018.12.015

Jayaram, N., Usvyat, D., and R Martin, A. C. (2016). Evaluating Tools for Transcription Factor Binding Site Prediction. BMC Bioinformatics 17, 547. doi:10.1186/s12859-016-1298-9

Jiao, Y., and Du, P. (2016). Performance Measures in Evaluating Machine Learning Based Bioinformatics Predictors for Classifications. Quantitative Biol. 4, 320-330. doi:10.1007/s40484-016-0081-2

Johnson, D. S., Mortazavi, A., Myers, R. M., and Wold, B. (2007). Genome-wide Mapping of In Vivo Protein-DNA Interactions. Science 316, 1497-1502. doi:10. 1126/science.1141319

Jolma, A., Yan, J., Whitington, T., Toivonen, J., Nitta, K. R., Rastas, P., et al. (2013). DNA-binding Specificities of Human Transcription Factors. Cell 152, 327-339. doi:10.1016/j.cell.2012.12.009

Jurtz, V. I., Johansen, A. R., Nielsen, M., Almagro Armenteros, J. J., Nielsen, H., Sønderby, C. K., et al. (2017). An Introduction to Deep Learning on Biological Sequence Data: Examples and Solutions. Bioinformatics 33, 3685-3690. doi:10. 1093/bioinformatics/btx531

Kawaguchi, R. K., Tang, Z., Fischer, S., Tripathy, R., Koo, P. K., and Gillis, J. (2021). Exploiting Marker Genes for Robust Classification and Characterization of Single-Cell Chromatin Accessibility. Cold Spring Harbor, NY: bioRxiv. doi:10.1101/2021.04.01.438068

Keilwagen, J., and Grau, J. (2015). Varying Levels of Complexity in Transcription Factor Binding Motifs. Nucleic Acids Res. 43, e119. doi:10.1093/nar/gkv577

Keilwagen, J., Posch, S., and Grau, J. (2019). Accurate Prediction of Cell Typespecific Transcription Factor Binding. Genome Biol. 20, 9. doi:10.1186/s13059018-1614-y 
Kelley, D. R. (2020). Cross-species Regulatory Sequence Activity Prediction. Plos Comput. Biol. 16, e1008050. doi:10.1371/journal.pcbi.1008050

Kelley, D. R., Reshef, Y. A., Bileschi, M., Belanger, D., McLean, C. Y., and Snoek, J. (2018). Sequential Regulatory Activity Prediction across Chromosomes with Convolutional Neural Networks. Genome Res. 28, 739-750. doi:10.1101/gr. 227819.117

Kelley, D. R., Snoek, J., and Rinn, J. L. Basset. (2016). Learning the Regulatory Code of the Accessible Genome with Deep Convolutional Neural Networks. Genome Res. 26, 990-999. doi:10.1101/gr.200535.115

Khan, A., Fornes, O., Stigliani, A., Gheorghe, M., Castro-Mondragon, J. A., van der Lee, R., et al. (2018). JASPAR 2018: Update of the Open-Access Database of Transcription Factor Binding Profiles and its Web Framework. Nucleic Acids Res. 46, D1284. doi:10.1093/nar/gkx1188

Killoran, N., Lee, L. J., Delong, A., Duvenaud, D., and Frey, B. J. (2017). Generating and Designing DNA with Deep Generative Models. Ithaca, NY: arXiv [cs.LG].

Kim, T. H., Abdullaev, Z. K., Smith, A. D., Ching, K. A., Loukinov, D. I., Green, R. D., et al. (2007). Analysis of the Vertebrate Insulator Protein CTCF-Binding Sites in the Human Genome. Cell 128, 1231-1245. doi:10.1016/j.cell.2006.12.048

Koo, P. K., Anand, P., Paul, S. B., and Eddy, S. R. (2018). Inferring SequenceStructure Preferences of Rna-Binding Proteins with Convolutional Residual Networks. Cold Spring Harbor, NY: BioRxiv. doi:10.1101/418459

Koo, P. K., and Eddy, S. R. (2019). Representation Learning of Genomic Sequence Motifs with Convolutional Neural Networks. Plos Comput. Biol. 15, e1007560. doi:10.1371/journal.pcbi.1007560

Koo, P. K., and Ploenzke, M. (2020). Deep Learning for Inferring Transcription Factor Binding Sites. Curr. Opin. Syst. Biol. 19, 16-23. doi:10.1016/j.coisb.2020.04.001

Koo, P. K., and Ploenzke, M. (2021). Improving Representations of Genomic Sequence Motifs in Convolutional Networks with Exponential Activations. Nat. Machine Intelligence 3, 258-266. doi:10.1038/s42256-020-00291-x

Koo, P. K., and Ploenzke, M. (2020). Interpreting Deep Neural Networks beyond Attribution Methods: Quantifying Global Importance of Genomic Features. Cold Spring Harbor, NY: bioRxiv.

Kopp, W., Monti, R., Tamburrini, A., Ohler, U., and Akalin, A. (2020). Deep Learning for Genomics Using Janggu. Nat. Commun. 11, 3488. doi:10.1038/ s41467-020-17155-y

Kotopka, B. J., and Smolke, C. D. (2020). Model-driven Generation of Artificial Yeast Promoters. Nat. Commun. 11, 2113. doi:10.1038/s41467-020-15977-4

Kudla, G., Murray, A. W., Tollervey, D., and Plotkin, J. B. (2009). Coding-sequence Determinants of Gene Expression in Escherichia coli. Science 324, 255-258. doi:10.1126/science.1170160

Kumar, A., and Bansal, M. (2017). Unveiling DNA Structural Features of Promoters Associated with Various Types of TSSs in Prokaryotic Transcriptomes and Their Role in Gene Expression. DNA Res. 24, 25-35. doi:10.1093/dnares/dsw045

Lahtvee, P.-J., Sánchez, B. J., Smialowska, A., Kasvandik, S., Elsemman, I. E., Gatto, F., et al. (2017). Absolute Quantification of Protein and mRNA Abundances Demonstrate Variability in Gene-specific Translation Efficiency in Yeast. Cell Syst 4, 495e5-504. doi:10.1016/j.cels.2017.03.003

Lanchantin, J., Singh, R., Wang, B., and Qi, Y. (2016). "DEEP MOTIF DASHBOARD: VISUALIZING AND UNDERSTANDING GENOMIC SEQUENCES USING DEEP NEURAL NETWORKS," in Biocomputing 2017 (World Scientific), 254-265.

LeCun, Y., Bengio, Y., and Hinton, G. (2015). Deep Learning. Nature 521, 436-444. doi:10.1038/nature14539

Lee, D., Gorkin, D. U., Baker, M., Strober, B. J., Asoni, A. L., McCallion, A. S., et al. (2015). A Method to Predict the Impact of Regulatory Variants from DNA Sequence. Nat. Genet. 47, 955-961. doi:10.1038/ng.3331

Lee, D., Karchin, R., and Beer, M. A. (2011). Discriminative Prediction of Mammalian Enhancers from DNA Sequence. Genome Res. 21, 2167-2180. doi:10.1101/gr.121905.111

Lee, D., Zhang, J., Liu, J., and Gerstein, M. (2020). Epigenome-based Splicing Prediction Using a Recurrent Neural Network. Plos Comput. Biol. 16, e1008006. doi:10.1371/journal.pcbi.1008006

Lee, T. I., and Young, R. A. (2013). Transcriptional Regulation and its Misregulation in Disease. Cell 152, 1237-1251. doi:10.1016/j.cell.2013.02.014

Leiby, N., Hossain, A., and Salis, H. M. (2020).Convolutional Neural Net Learns Promoter Sequence Features Driving Transcription Strength. Manchester, United Kingdom: EasyChair. doi:10.29007/8fmw
Leman, R., Gaildrat, P., Le Gac, G., Ka, C., Fichou, Y., Audrezet, M.-P., et al. (2018). Novel Diagnostic Tool for Prediction of Variant Spliceogenicity Derived from a Set of 395 Combined In Silico/In Vitro Studies: an International Collaborative Effort. Nucleic Acids Res. 46, 7913-7923. doi:10.1093/nar/gky372

Leman, R., Tubeuf, H., Raad, S., Tournier, I., Derambure, C., Lanos, R., et al. (2020). Assessment of branch point Prediction Tools to Predict Physiological branch Points and Their Alteration by Variants. BMC Genomics 21, 86. doi:10.1186/ s12864-020-6484-5

Leppek, K., Das, R., and Barna, M. (2018). Functional 5' UTR mRNA Structures in Eukaryotic Translation Regulation and How to Find Them. Nat. Rev. Mol. Cel Biol. 19, 158-174. doi:10.1038/nrm.2017.103

Levo, M., and Segal, E. (2014). In Pursuit of Design Principles of Regulatory Sequences. Nat. Rev. Genet. 15, 453-468. doi:10.1038/nrg3684

Levo, M., Zalckvar, E., Sharon, E., Dantas Machado, A. C., Kalma, Y., LotamPompan, M., et al. (2015). Unraveling Determinants of Transcription Factor Binding outside the Core Binding Site. Genome Res. 25, 1018-1029. doi:10. 1101/gr.185033.114

Li, G., Zrimec, Jan., Ji, Boyang., Geng, Jun., Larsbrink, Johan., Zelezniak, Aleksej., et al. (2019). Performance of Regression Models as a Function of experiment Noise. arXiv [q-bio.BM].

Li, J. J., Chew, G.-L., and Biggin, M. D. (2017). Quantitating Translational Control: mRNA Abundance-dependent and Independent Contributions and the mRNA Sequences that Specify Them. Nucleic Acids Res. 45, 11821-11836. doi:10.1093/ nar/gkx 898

Li, J. J., Chew, G.-L., and Biggin, M. D. (2019). Quantitative Principles of CisTranslational Control by General mRNA Sequence Features in Eukaryotes. Genome Biol. 20, 162. doi:10.1186/s13059-019-1761-9

Li, J., Liang, Q., Song, W., and Marchisio, M. A. (2017). Nucleotides Upstream of the Kozak Sequence Strongly Influence Gene Expression in the Yeast $S$. cerevisiae. J. Biol. Eng. 11, 25. doi:10.1186/s13036-017-0068-1

Lin, H., Deng, E. Z., Ding, H., Chen, W., and Chou, K. C. (2014). iPro54-PseKNC: a Sequence-Based Predictor for Identifying Sigma-54 Promoters in Prokaryote with Pseudo K-Tuple Nucleotide Composition. Nucleic Acids Res. 42, 12961-12972. doi:10.1093/nar/gku1019

Liu, Y., Barr, K., and Reinitz, J. (2020). Fully Interpretable Deep Learning Model of Transcriptional Control. Bioinformatics 36, i499-i507. doi:10.1093/ bioinformatics/btaa506

Liu, Y., Beyer, A., and Aebersold, R. (2016). On the Dependency of Cellular Protein Levels on mRNA Abundance. Cell 165, 535-550. doi:10.1016/j.cell. 2016.03.014

Lu, R., and Rogan, P. K. (2018). Transcription Factor Binding Site Clusters Identify Target Genes with Similar Tissue-wide Expression and Buffer against Mutations. F1000Res 7, 1933. doi:10.12688/f1000research.17363.1

Lubliner, S., Regev, I., Lotan-Pompan, M., Edelheit, S., Weinberger, A., and Segal, E. (2015). Core Promoter Sequence in Yeast Is a Major Determinant of Expression Level. Genome Res. 25, 1008-1017. doi:10.1101/gr.188193.114

Lundberg, S., and Lee, S. I. (2017). A Unified Approach to Interpreting Model Predictions. Ithaca, NY: arXiv [cs.AI].

Ma, J., Yu, M. K., Fong, S., Ono, K., Sage, E., Demchak, B., et al. (2018). Using Deep Learning to Model the Hierarchical Structure and Function of a Cell. Nat. Methods 15, 290-298. doi:10.1038/nmeth.4627

Marcovitz, A., and Levy, Y. (2013). Weak Frustration Regulates Sliding and Binding Kinetics on Rugged Protein-DNA Landscapes. J. Phys. Chem. B 117, 13005-13014. doi:10.1021/jp402296d

Martin, V., Zhao, J., Afek, A., Mielko, Z., and Gordân, R. (2019). QBiC-Pred: Quantitative Predictions of Transcription Factor Binding Changes Due to Sequence Variants. Nucleic Acids Res. 47, W127-W135. doi:10.1093/nar/gkz363

Maslova, A., Ramirez, R. N., Ma, K., Schmutz, H., Wang, C., Fox, C., et al. (2020). Deep Learning of Immune Cell Differentiation. Proc. Natl. Acad. Sci. U. S. A. 117, 25655-25666. doi:10.1073/pnas.2011795117

Mathelier, A., Xin, B., Chiu, T.-P., Yang, L., Rohs, R., and Wasserman, W. W. (2016). DNA Shape Features Improve Transcription Factor Binding Site Predictions In Vivo. Cel Syst 3, 278-286. doi:10.1016/j.cels.2016.07.001e4

Mayr, C. (2017). Regulation by 3'-Untranslated Regions. Annu. Rev. Genet. 51, 171-194. doi:10.1146/annurev-genet-120116-024704

Mercer, T. R., Clark, M. B., Andersen, S. B., Brunck, M. E., Haerty, W., Crawford, J., et al. (2015). Genome-wide Discovery of Human Splicing Branchpoints. Genome Res. 25, 290-303. doi:10.1101/gr.182899.114 
Meysman, P., Marchal, K., and Engelen, K. (2012). DNA Structural Properties in the Classification of Genomic Transcription Regulation Elements. Bioinform. Biol. Insights 6, 155-168. doi:10.4137/BBI.S9426

Mhaskar, H., Liao, Q., and Poggio, T. (2017). "When and Why Are Deep Networks Better Than Shallow Ones?," in AAAI, 31.

Millar, A. H., Heazlewood, J. L., Giglione, C., Holdsworth, M. J., Bachmair, A., and Schulze, W. X. (2019). The Scope, Functions, and Dynamics of Posttranslational Protein Modifications. Annu. Rev. Plant Biol. 70, 119-151. doi:10.1146/annurev-arplant-050718-100211

Miller, J. L., and Grant, P. A. (2013). The Role of DNA Methylation and Histone Modifications in Transcriptional Regulation in Humans. Subcell. Biochem. 61, 289-317. doi:10.1007/978-94-007-4525-4_13

Min, X., Zeng, W., Chen, S., Chen, N., Chen, T., and Jiang, R. (2017). Predicting Enhancers with Deep Convolutional Neural Networks. BMC Bioinformatics 18, 478. doi:10.1186/s12859-017-1878-3

Mittal, P., Brindle, J., Stephen, J., Plotkin, J. B., and Kudla, G. (2018). Codon Usage Influences Fitness through RNA Toxicity. Proc. Natl. Acad. Sci. U. S. A. 115, 8639-8644. doi:10.1073/pnas.1810022115

Montavon, G., Samek, W., and Müller, K.-R. (2018). Methods for Interpreting and Understanding Deep Neural Networks. Digit. Signal. Process. 73, 1-15. doi:10. 1016/j.dsp.2017.10.011

Moqtaderi, Z., Geisberg, J. V., Jin, Y., Fan, X., and Struhl, K. (2013). Species-specific Factors Mediate Extensive Heterogeneity of mRNA 3' Ends in Yeasts. Proc. Natl. Acad. Sci. U. S. A. 110, 11073-11078. doi:10.1073/pnas.1309384110

Mora, A., Sandve, G. K., Gabrielsen, O. S., and Eskeland, R. (2016). The Loop: Promoter-Enhancer Interactions and Bioinformatics. Brief. Bioinform. 17, 980-995. doi:10.1093/bib/bbv097

Morse, N. J., Gopal, M. R., Wagner, J. M., and Alper, H. S. (2017). Yeast Terminator Function Can Be Modulated and Designed on the Basis of Predictions of Nucleosome Occupancy. ACS Synth. Biol. 6, 2086-2095. doi:10.1021/acssynbio.7b00138

Movva, R., Greenside, P., Marinov, G. K., Nair, S., Shrikumar, A., and Kundaje, A. (2019). Deciphering Regulatory DNA Sequences and Noncoding Genetic Variants Using Neural Network Models of Massively Parallel Reporter Assays. PLoS One 14, e0218073. doi:10.1371/journal.pone.0218073

Nagy, G., and Nagy, L. (2020). Motif Grammar: The Basis of the Language of Gene Expression. Comput. Struct. Biotechnol. J. 18, 2026-2032. doi:10.1016/j.csbj. 2020.07.007

Naidoo, T., Sjödin, P., Schlebusch, C., and Jakobsson, M. (2018). Patterns of Variation in Cis-Regulatory Regions: Examining Evidence of Purifying Selection. BMC Genomics 19, 95. doi:10.1186/s12864-017-4422-y

Nakagawa, S., Niimura, Y., Gojobori, T., Tanaka, H., and Miura, K.-I. (2008). Diversity of Preferred Nucleotide Sequences Around the Translation Initiation Codon in Eukaryote Genomes. Nucleic Acids Res. 36, 861-871. doi:10.1093/nar/gkm1102

Naulaerts, S., Meysman, P., Bittremieux, W., Vu, T. N., Vanden Berghe, W., Goethals, B., et al. (2015). A Primer to Frequent Itemset Mining for Bioinformatics. Brief. Bioinform. 16, 216-231. doi:10.1093/bib/bbt074

Nazari, I., Tayara, H., and Chong, K. T. (2019). Branch Point Selection in RNA Splicing Using Deep Learning. IEEE Access 7, 1800-1807. doi:10.1109/access. 2018.2886569

Neymotin, B., Ettorre, V., and Gresham, D. (2016). Multiple Transcript Properties Related to Translation Affect mRNA Degradation Rates in Saccharomyces cerevisiae. G 6, 3475-3483. doi:10.1534/g3.116.032276

Nielsen, J., and Keasling, J. D. (2016). Engineering Cellular Metabolism. Cell 164, 1185-1197. doi:10.1016/j.cell.2016.02.004

Nielsen, J. (2017). Systems Biology of Metabolism. Annu. Rev. Biochem. 86, 245-275. doi:10.1146/annurev-biochem-061516-044757

Niu, X., Yang, K., Zhang, G., Yang, Z., and Hu, X. (2019). A Pretraining-Retraining Strategy of Deep Learning Improves Cell-specific Enhancer Predictions. Front. Genet. 10, 1305. doi:10.3389/fgene.2019.01305

Omotajo, D., Tate, T., Cho, H., and Choudhary, M. (2015). Distribution and Diversity of Ribosome Binding Sites in Prokaryotic Genomes. BMC Genomics 16, 604. doi:10.1186/s12864-015-1808-6

Paggi, J. M., and Bejerano, G. (2018). A Sequence-Based, Deep Learning Model Accurately Predicts RNA Splicing Branchpoints. RNA 24, 1647-1658. doi:10. 1261/rna.066290.118

Park, C., Qian, W., and Zhang, J. (2012). Genomic Evidence for Elevated Mutation Rates in Highly Expressed Genes. EMBO Rep. 13, 1123-1129. doi:10.1038/ embor.2012.165
Park, S., Koh, Y., Jeon, H., Kim, H., Yeo, Y., and Kang, J. (2020). Enhancing the Interpretability of Transcription Factor Binding Site Prediction Using Attention Mechanism. Sci. Rep. 10, 13413. doi:10.1038/s41598-020-70218-4

Pennacchio, L. A., Bickmore, W., Dean, A., Nobrega, M. A., and Bejerano, G. (2013). Enhancers: Five Essential Questions. Nat. Rev. Genet. 14, 288-295. doi:10.1038/nrg3458

Peters, J. M., Mooney, R. A., Kuan, P. F., Rowland, J. L., Keles, S., and Landick, R. (2009). Rho Directs Widespread Termination of Intragenic and Stable RNA Transcription. Proc. Natl. Acad. Sci. U. S. A. 106, 15406-15411. doi:10.1073/ pnas.0903846106

Playe, B., and Stoven, V. (2020). Evaluation of Deep and Shallow Learning Methods in Chemogenomics for the Prediction of Drugs Specificity. J. Cheminform. 12, 11. doi:10.1186/s13321-020-0413-0

Plotkin, J. B., and Kudla, G. (2011). Synonymous but Not the Same: the Causes and Consequences of Codon Bias. Nat. Rev. Genet. 12, 32-42. doi:10.1038/nrg2899

Presnyak, V., Alhusaini, N., Chen, Y.-H., Martin, S., Morris, N., Kline, N., et al. (2015). Codon Optimality Is a Major Determinant of mRNA Stability. Cell 160, 1111-1124. doi:10.1016/j.cell.2015.02.029

Puigbò, P., Guzmán, E., Romeu, A., and Garcia-Vallvé, S. (2007). OPTIMIZER: a Web Server for Optimizing the Codon Usage of DNA Sequences. Nucleic Acids Res. 35, W126-W131. doi:10.1093/nar/gkm219

Quang, D., and Xie, X. Dan. Q. (2016). A Hybrid Convolutional and Recurrent Deep Neural Network for Quantifying the Function of DNA Sequences. Nucleic Acids Res. 44, e107. doi:10.1093/nar/gkw226

Rao, R., Bhattacharya, N., Thomas, N., Duan, Y., Chen, X., Canny, J., et al. (2019). Evaluating Protein Transfer Learning with TAPE. Adv. Neural Inf. Process. Syst. 32, 9689-9701.

Re, A., Joshi, T., Kulberkyte, E., Morris, Q., and Workman, C. T. (2014). RNAprotein Interactions: an Overview. Methods Mol. Biol. 1097, 491-521. doi:10. 1007/978-1-62703-709-9_23

Redden, H., and Alper, H. S. (2015). The Development and Characterization of Synthetic Minimal Yeast Promoters. Nat. Commun. 6, 7810. doi:10.1038/ ncomms 8810

Rehbein, P., Berz, J., Kreisel, P., and Schwalbe, H. (2019). 'CodonWizard' - an Intuitive Software Tool with Graphical User Interface for Customizable Codon Optimization in Protein Expression Efforts. Protein Expr. Purif. 160, 84-93. doi:10.1016/j.pep.2019.03.018

Ren, G.-X., Guo, X.-P., and Sun, Y.-C. (2017). Regulatory 3' Untranslated Regions of Bacterial mRNAs. Front. Microbiol. 8, 1276. doi:10.3389/fmicb.2017.01276

Repecka, D., Jauniskis, V., Karpus, L., Rembeza, E., Rokaitis, I., Zrimec, J., et al. (2021). Expanding Functional Protein Sequence Spaces Using Generative Adversarial Networks. Nat. Machine Intelligence 3, 324-333. doi:10.1038/ s42256-021-00310-5

Richardson, S. M., Wheelan, S. J., Yarrington, R. M., and Boeke, J. D. (2006). GeneDesign: Rapid, Automated Design of Multikilobase Synthetic Genes. Genome Res. 16, 550-556. doi:10.1101/gr.4431306

Roberts, J. W. (2019). Mechanisms of Bacterial Transcription Termination. J. Mol. Biol. 431, 4030-4039. doi:10.1016/j.jmb.2019.04.003

Rohs, R., Jin, X., West, S. M., Joshi, R., Honig, B., and Mann, R. S. (2010). Origins of Specificity in Protein-DNA Recognition. Annu. Rev. Biochem. 79, 233-269. doi:10.1146/annurev-biochem-060408-091030

Rohs, R., West, S. M., Sosinsky, A., Liu, P., Mann, R. S., and Honig, B. (2009). The Role of DNA Shape in Protein-DNA Recognition. Nature 461, 1248-1253. doi:10.1038/nature08473

Rudin, C. (2019). Stop Explaining Black Box Machine Learning Models for High Stakes Decisions and Use Interpretable Models Instead. Nat. Machine Intelligence 1, 206-215. doi:10.1038/s42256-019-0048-x

Saier, M. H., Jr. (2019). Understanding the Genetic Code. J. Bacteriol. 201. doi:10. 1128/JB.00091-19

Salis, H. M., Mirsky, E. A., and Voigt, C. A. (2009). Automated Design of Synthetic Ribosome Binding Sites to Control Protein Expression. Nat. Biotechnol. 27, 946-950. doi:10.1038/nbt.1568

Salis, H. M. (2011). The Ribosome Binding Site Calculator. Methods Enzymol. 498, 19-42. doi:10.1016/b978-0-12-385120-8.00002-4

Sample, P. J., Wang, B., Reid, D. W., Presnyak, V., McFadyen, I. J., Morris, D. R., et al. (2019). Human 5' UTR Design and Variant Effect Prediction from a Massively Parallel Translation Assay. Nat. Biotechnol. 37, 803-809. doi:10.1038/ s41587-019-0164-5 
SantaLucia, J., Jr. (1998). A Unified View of Polymer, Dumbbell, and Oligonucleotide DNA Nearest-Neighbor Thermodynamics. Proc. Natl. Acad. Sci. U. S. A. 95, 1460-1465. doi:10.1073/pnas.95.4.1460

Schwanhäusser, B., Busse, D., Li, N., Dittmar, G., Schuchhardt, J., Wolf, J., et al. (2011). Global Quantification of Mammalian Gene Expression Control. Nature 473, 337-342. doi:10.1038/nature10098

Segal, E., and Widom, J. (2009). From DNA Sequence to Transcriptional Behaviour: a Quantitative Approach. Nat. Rev. Genet. 10, 443-456. doi:10.1038/nrg2591

Shalem, O., Sharon, E., Lubliner, S., Regev, I., Lotan-Pompan, M., Yakhini, Z., et al. (2015). Systematic Dissection of the Sequence Determinants of Gene 3'end Mediated Expression Control. Plos Genet. 11, e1005147. doi:10.1371/journal. pgen.1005147

Sharon, E., Kalma, Y., Sharp, A., Raveh-Sadka, T., Levo, M., Zeevi, D., et al. (2012). Inferring Gene Regulatory Logic from High-Throughput Measurements of Thousands of Systematically Designed Promoters. Nat. Biotechnol. 30, 521-530. doi:10.1038/nbt.2205

Sharp, P. M., and Li, W. H. (1987). The Codon Adaptation Index--a Measure of Directional Synonymous Codon Usage Bias, and its Potential Applications. Nucleic Acids Res. 15, 1281-1295. doi:10.1093/nar/15.3.1281

Shine, J., and Dalgarno, L. (1975). Determinant of Cistron Specificity in Bacterial Ribosomes. Nature 254, 34-38. doi:10.1038/254034a0

Shlyueva, D., Stampfel, G., and Stark, A. (2014). Transcriptional Enhancers: from Properties to Genome-wide Predictions. Nat. Rev. Genet. 15, 272-286. doi:10. $1038 / \operatorname{nrg} 3682$

Shrikumar, A., Greenside, P., and Kundaje, A. (2017). Learning Important Features through Propagating Activation Differences. Ithaca, NY: arXiv [cs.CV].

Shrikumar, A., Tian, Katherine., Avsec, Žiga., Shcherbina, Anna., Banerjee, Abhimanyu., Sharmin, Mahfuza., et al. (2018). Technical Note on Transcription Factor Motif Discovery from Importance Scores (TF-MoDISco) Version 0.5.6.5. Ithaca, NY: arXiv [cs.LG].

Siggers, T., and Gordân, R. (2014). Protein-DNA Binding: Complexities and MultiProtein Codes. Nucleic Acids Res. 42, 2099-2111. doi:10.1093/nar/gkt1112

Signal, B., Gloss, B. S., Dinger, M. E., and Mercer, T. R. (2018). Machine Learning Annotation of Human Branchpoints. Bioinformatics 34, 920-927. doi:10.1093/ bioinformatics/btx688

Simonyan, K., Vedaldi, A., and Zisserman, A. (2013). Deep inside Convolutional Networks: Visualising Image Classification Models and Saliency Maps. Ithaca, NY: arXiv [cs.CV].

Singh, R., Lanchantin, J., Robins, G., and Qi, Y. Deep. Chrome. (2016). Deeplearning for Predicting Gene Expression from Histone Modifications. Bioinformatics 32, i639-i648. doi:10.1093/bioinformatics/btw427

Singh, R., Lanchantin, J., Sekhon, A., and Qi, Y. (2017). Attend and Predict: Understanding Gene Regulation by Selective Attention on Chromatin. Adv. Neural Inf. Process. Syst. 30, 6785-6795.

Singh, S., Yang, Y., Póczos, B., and Ma, J. (2019). Predicting Enhancer-Promoter Interaction from Genomic Sequence with Deep Neural Networks. Quantitative Biol. 7, 122-137. doi:10.1007/s40484-019-0154-0

Slattery, M., Zhou, T., Yang, L., Dantas Machado, A. C., Gordân, R., and Rohs, R. (2014). Absence of a Simple Code: How Transcription Factors Read the Genome. Trends Biochem. Sci. 39, 381-399. doi:10.1016/j.tibs.2014.07.002

Song, L., and Crawford, G. E. (2010). DNase-Seq: A High-Resolution Technique for Mapping Active Gene Regulatory Elements across the Genome from Mammalian Cells. Cold Spring Harbor, NY: Cold Spring Harb. Protoc. doi:10.1101/pdb.prot5384

Sonnenburg, S., Schweikert, G., Philips, P., Behr, J., and Rätsch, G. (2007). Accurate Splice Site Prediction Using Support Vector Machines. BMC Bioinformatics 8 (Suppl. 10), S7. doi:10.1186/1471-2105-8-S10-S7

Stormo, G. D. (2000). DNA Binding Sites: Representation and Discovery. Bioinformatics 16, 16-23. doi:10.1093/bioinformatics/16.1.16

Strokach, A., Becerra, D., Corbi-Verge, C., Perez-Riba, A., and Kim, P. M. (2020). Fast and Flexible Protein Design Using Deep Graph Neural Networks. Cel Syst 11, 402e4-411. doi:10.1016/j.cels.2020.08.016

Strubell, E., Verga, P., Belanger, D., and McCallum, A. (2017). Fast and Accurate Sequence Labeling with Iterated Dilated Convolutions.

Struhl, K., and Segal, E. (2013). Determinants of Nucleosome Positioning. Nat. Struct. Mol. Biol. 20, 267-273. doi:10.1038/nsmb.2506

Tafvizi, A., Mirny, L. A., and van Oijen, A. M. (2011). Dancing on DNA: Kinetic Aspects of Search Processes on DNA. Chemphyschem 12, 1481-1489. doi:10. 1002/cphc.201100112
Tang, L., Hill, M. C., Wang, J., Wang, J., Martin, J. F., and Li, M. (2020). Predicting Unrecognized Enhancer-Mediated Genome Topology by an Ensemble Machine Learning Model. Genome Res. 30, 1835-1845. doi:10.1101/gr. 264606.120

Tareen, A., and Kinney, J. B. (2019). Biophysical Models of Cis-Regulation as Interpretable Neural Networks. Ithaca, NY: arXiv [q-bio.MN].

Terai, G., and Asai, K. (2020). Improving the Prediction Accuracy of Protein Abundance in Escherichia coli Using mRNA Accessibility. Nucleic Acids Res. 48, e81. doi:10.1093/nar/gkaa481

Tian, B., and Manley, J. L. (2017). Alternative Polyadenylation of mRNA Precursors. Nat. Rev. Mol. Cel Biol. 18, 18-30. doi:10.1038/nrm.2016.116

Tian, Q., Zou, J., Tang, J., Fang, Y., Yu, Z., and Fan, S. (2019). MRCNN: a Deep Learning Model for Regression of Genome-wide DNA Methylation. BMC Genomics 20, 192. doi:10.1186/s12864-019-5488-5

Tirosh, I., Reikhav, S., Levy, A. A., and Barkai, N. (2009). A Yeast Hybrid Provides Insight into the Evolution of Gene Expression Regulation. Science 324, 659-662. doi:10.1126/science.1169766

Trabelsi, A., Chaabane, M., and Ben-Hur, A. (2019). Comprehensive Evaluation of Deep Learning Architectures for Prediction of DNA/RNA Sequence Binding Specificities. Bioinformatics 35, i269-i277. doi:10.1093/ bioinformatics/btz339

Trösemeier, J.-H., Rudorf, S., Loessner, H., Hofner, B., Reuter, A., Schulenborg, T., et al. (2019). Optimizing the Dynamics of Protein Expression. Sci. Rep. 9, 7511. doi:10.1038/s41598-019-43857-5

Trotta, E. (2013). Selection on Codon Bias in Yeast: a Transcriptional Hypothesis. Nucleic Acids Res. 41, 9382-9395. doi:10.1093/nar/gkt740

Tsai, Z. T.-Y., Shiu, S.-H., and Tsai, H.-K. (2015). Contribution of Sequence Motif, Chromatin State, and DNA Structure Features to Predictive Models of Transcription Factor Binding in Yeast. Plos Comput. Biol. 11, e1004418. doi:10.1371/journal.pcbi.1004418

Tuller, T., Waldman, Y. Y., Kupiec, M., and Ruppin, E. (2010). Translation Efficiency Is Determined by Both Codon Bias and Folding Energy. Proc. Natl. Acad. Sci. U. S. A. 107, 3645-3650. doi:10.1073/pnas.0909910107

Tunney, R., McGlincy, N. J., Graham, M. E., Naddaf, N., Pachter, L., and Lareau, L. F. (2018). Accurate Design of Translational Output by a Neural Network Model of Ribosome Distribution. Nat. Struct. Mol. Biol. 25, 577-582. doi:10.1038/ s41594-018-0080-2

Ullah, F., and Ben-Hur, A. (2020). A Self-Attention Model for Inferring Cooperativity between Regulatory Features. Cold Spring Harbor, NY: Cold Spring Harbor Laboratory. doi:10.1101/2020.01.31.927996

Urtecho, G., Tripp, A. D., Insigne, K. D., Kim, H., and Kosuri, S. (2019). Systematic Dissection of Sequence Elements Controlling 670 Promoters Using a Genomically Encoded Multiplexed Reporter Assay in Escherichia coli. Biochemistry 58, 1539-1551. doi:10.1021/acs.biochem.7b01069

Van Brempt, M., Clauwaert, J., Mey, F., Stock, M., Maertens, J., Waegeman, W., et al. (2020). Predictive Design of Sigma Factor-specific Promoters. Nat. Commun. 11, 5822. doi:10.1038/s41467-020-19446-w

Vaswani, A., Shazeer, Noam., Parmar, Niki., Uszkoreit, Jakob., Jones, Llion., Gomez, Aidan. N., et al. (2017). Attention Is All You Need. arXiv [cs.CL].

Vig, J., Madani, Ali., Varshney, Lav. R., Xiong, Caiming., Socher, Richard., and Rajani, Nazneen. Fatema. (2020). BERTology Meets Biology: Interpreting Attention in Protein Language Models. Ithaca, NY: arXiv [cs.CL].

Visel, A., Blow, M. J., Li, Z., Zhang, T., Akiyama, J. A., Holt, A., et al. (2009). ChIPseq Accurately Predicts Tissue-specific Activity of Enhancers. Nature 457, 854-858. doi:10.1038/nature07730

Vogel, C., de Sousa Abreu, R., Ko, D., Le, S. Y., Shapiro, B. A., Burns, S. C., et al. (2010). Sequence Signatures and mRNA Concentration Can Explain TwoThirds of Protein Abundance Variation in a Human Cell Line. Mol. Syst. Biol. 6 , 400. doi: $10.1038 / \mathrm{msb} .2010 .59$

Wang, M., Tai, C., E, W., and Wei, L. De. Fine. (2018). Deep Convolutional Neural Networks Accurately Quantify Intensities of Transcription Factor-DNA Binding and Facilitate Evaluation of Functional Non-coding Variants. Nucleic Acids Res. 46, e69. doi:10.1093/nar/gky215

Wang, R., Wang, Z., Wang, J., and Li, S. (2019). SpliceFinder: Ab Initio Prediction of Splice Sites Using Convolutional Neural Network. BMC Bioinformatics 20, 652. doi:10.1186/s12859-019-3306-3

Wang, X., Girshick, R., Gupta, A., and He, K. (2017). Non-local Neural Networks. arXiv [cs.CV]. 
Wang, H., Cimen, E., Singh, N., and Buckler, E. (2020). Deep Learning for Plant Genomics and Crop Improvement. Curr. Opin. Plant Biol. 54, 34-41. doi:10. 1016/j.pbi.2019.12.010

Wang, Y., Wang, H., Wei, L., Li, S., Liu, L., and Wang, X. (2020). Synthetic Promoter Design in Escherichia coli Based on a Deep Generative Network. Nucleic Acids Res. 48, 6403-6412. doi:10.1093/nar/gkaa325

Wang, Z., Gerstein, M., and Snyder, M. (2009). RNA-seq: a Revolutionary Tool for Transcriptomics. Nat. Rev. Genet. 10, 57-63. doi:10.1038/nrg2484

Washburn, J. D., Mejia-Guerra, M. K., Ramstein, G., Kremling, K. A., Valluru, R., Buckler, E. S., et al. (2019). Evolutionarily Informed Deep Learning Methods for Predicting Relative Transcript Abundance from DNA Sequence. Proc. Natl. Acad. Sci. U. S. A. 116, 5542-5549. doi:10.1073/pnas.1814551116

Watson, J. D., Baker, T. A., Bell, S. P., Gann, A., Levine, M., and Losick, R. (2008). Molecular Biology of the Gene. 6th. ed.. San Francisco, CA: Pearson/Benjamin Cummings.

Way, G. P., and Greene, C. S. (2018). Extracting a Biologically Relevant Latent Space from Cancer Transcriptomes with Variational Autoencoders. Pac. Symp. Biocomput. 23, 80-91.

Webb, S. (2018). Deep Learning for Biology. Nature 554, 555-557. doi:10.1038/ d41586-018-02174-z

Weenink, T., van der Hilst, J., McKiernan, R. M., and Ellis, T. (2018). Design of RNA Hairpin Modules that Predictably Tune Translation in Yeast. Synth. Biol. 3, ysy019. doi:10.1093/synbio/ysy019

Weirauch, M. T., au, fnm., Cote, A., Norel, R., Annala, M., Zhao, Y., et al. (2013). Evaluation of Methods for Modeling Transcription Factor Sequence Specificity. Nat. Biotechnol. 31, 126-134. doi:10.1038/nbt.2486

Whitaker, J. W., Chen, Z., and Wang, W. (2015). Predicting the Human Epigenome from DNA Motifs. Nat. Methods 12 (), 265-272. 7 p following 272. doi:10.1038/nmeth.3065

Wilkinson, M. E., Charenton, C., and Nagai, K. (2020). RNA Splicing by the Spliceosome. Аnnu. Rev. Biochem. 89, 359-388. doi:10.1146/annurev-biochem-091719-064225

Wittkopp, P. J., Haerum, B. K., and Clark, A. G. (2004). Evolutionary Changes in Cis and Trans Gene Regulation. Nature 430, 85-88. doi:10.1038/nature02698

Wittkopp, P. J., and Kalay, G. (2011). Cis-regulatory Elements: Molecular Mechanisms and Evolutionary Processes Underlying Divergence. Nat. Rev. Genet. 13, 59-69. doi:10.1038/nrg3095

Xie, R., Wen, J., Quitadamo, A., Cheng, J., and Shi, X. (2017). A Deep AutoEncoder Model for Gene Expression Prediction. BMC Genomics 18, 845. doi:10. 1186/s12864-017-4226-0

Xu, Y., Wang, Y., Luo, J., Zhao, W., and Zhou, X. (2017). Deep Learning of the Splicing (Epi)genetic Code Reveals a Novel Candidate Mechanism Linking Histone Modifications to ESC Fate Decision. Nucleic Acids Res. 45, 12100-12112. doi:10.1093/nar/gkx870

Yang, D. K., Goldman, S. L., Weinstein, E., and Marks, D. (2019). "Generative Models for Codon Prediction and Optimization," in Machine Learning in Computational Biology.

Yang, L., Orenstein, Y., Jolma, A., Yin, Y., Taipale, J., Shamir, R., et al. (2017). Transcription Factor Family-specific DNA Shape Readout Revealed by Quantitative Specificity Models. Mol. Syst. Biol. 13, 910. doi:10.15252/msb.20167238

Yu, F., and Koltun, V. (2015). Multi-Scale Context Aggregation by Dilated Convolutions. arXiv [cs.CV].

Yu, M., Guo, W., Wang, Q., and Chen, J. Q. (2019). Widespread Positive Selection for mRNA Secondary Structure at Synonymous Sites in Domesticated Yeast. bioRxiv.

Zelezniak, A., Vowinckel, J., Capuano, F., Messner, C. B., Demichev, V., Polowsky, N., et al. (2018). Machine Learning Predicts the Yeast Metabolome from the Quantitative Proteome of Kinase Knockouts. Cel Syst 7, 269-283. e6. doi:10. 1016/j.cels.2018.08.001

Zeng, H., Edwards, M. D., Liu, G., and Gifford, D. K. (2016). Convolutional Neural Network Architectures for Predicting DNA-Protein Binding. Bioinformatics 32, i121-i127. doi:10.1093/bioinformatics/btw255

Zhang, Q., Fan, X., Wang, Y., Sun, M.-a., Shao, J., and Guo, D. (2017). BPP: a Sequence-Based Algorithm for branch point Prediction. Bioinformatics 33, 3166-3172. doi:10.1093/bioinformatics/btx401

Zhang, S., Hu, H., Jiang, T., Zhang, L., and Zeng, J. T. I. T. E. R. (2017). Predicting Translation Initiation Sites by Deep Learning. Bioinformatics 33, i234-i242. doi:10.1093/bioinformatics/btx247

Zhang, S., Hu, H., Zhou, J., He, X., Jiang, T., and Zeng, J. (2017). Analysis of Ribosome Stalling and Translation Elongation Dynamics by Deep Learning. Cel Syst 5, 212-220. e6. doi:10.1016/j.cels.2017.08.004
Zhang, Y., Zhou, X., and Cai, X. (2020). Predicting Gene Expression from DNA Sequence Using Residual Neural Network. Cold Spring Harbor, NY: Cold Spring Harbor Laboratory. doi:10.1101/2020.06.21.163956

Zhang, Z., Pan, Z., Ying, Y., Xie, Z., Adhikari, S., Phillips, J., et al. (2019). Deeplearning Augmented RNA-Seq Analysis of Transcript Splicing. Nat. Methods 16, 307-310. doi:10.1038/s41592-019-0351-9

Zhao, B. S., Roundtree, I. A., and He, C. (2017). Post-transcriptional Gene Regulation by mRNA Modifications. Nat. Rev. Mol. Cel Biol. 18, 31-42. doi:10.1038/nrm.2016.132

Zhao, J., Hyman, L., and Moore, C. (1999). Formation of mRNA 3' Ends in Eukaryotes: Mechanism, Regulation, and Interrelationships with Other Steps in mRNA Synthesis. Microbiol. Mol. Biol. Rev. 63 (2), 405. doi:10.1128/MMBR.63.2.405-445.1999

Zhou, J., Theesfeld, C. L., Yao, K., Chen, K. M., Wong, A. K., and Troyanskaya, O. G. (2018). Deep Learning Sequence-Based Ab Initio Prediction of Variant Effects on Expression and Disease Risk. Nat. Genet. 50, 1171-1179. doi:10.1038/ s41588-018-0160-6

Zhou, J., and Troyanskaya, O. G. (2015). Predicting Effects of Noncoding Variants with Deep Learning-Based Sequence Model. Nat. Methods 12, 931-934. doi:10. 1038/nmeth.3547

Zhou, T., Shen, N., Yang, L., Abe, N., Horton, J., Mann, R. S., et al. (2015). Quantitative Modeling of Transcription Factor Binding Specificities Using DNA Shape. Proc. Natl. Acad. Sci. U. S. A. 112, 4654-4659. doi:10.1073/ pnas. 1422023112

Zhou, Z., Dang, Y., Zhou, M., Li, L., Yu, C.-h., Fu, J., et al. (2016). Codon Usage Is an Important Determinant of Gene Expression Levels Largely through its Effects on Transcription. Proc. Natl. Acad. Sci. U. S. A. 113, E6117-E6125. doi:10.1073/pnas.1606724113

Zhou, Z., Dang, Y., Zhou, M., Yuan, H., and Liu, Y. (2018). Codon Usage Biases Coevolve with Transcription Termination Machinery to Suppress Premature Cleavage and Polyadenylation. Elife 7, e33569. doi:10.7554/eLife.33569

Zicola, J., Liu, L., Tänzler, P., and Turck, F. (2019). Targeted DNA Methylation Represses Two Enhancers of FLOWERING LOCUS $\mathrm{T}$ in Arabidopsis thaliana. Nat. Plants 5, 300-307. doi:10.1038/s41477-019-0375-2

Zou, J., Huss, M., Abid, A., Mohammadi, P., Torkamani, A., and Telenti, A. (2019). A Primer on Deep Learning in Genomics. Nat. Genet. 51, 12-18. doi:10.1038/ s41588-018-0295-5

Zrimec, J., Börlin, C. S., Buric, F., Muhammad, A. S., Chen, R., Siewers, V., et al. (2020). Deep Learning Suggests that Gene Expression Is Encoded in All Parts of a Co-evolving Interacting Gene Regulatory Structure. Nat. Commun. 11, 6141. doi:10.1038/s41467-020-19921-4

Zrimec, J., and Lapanje, A. (2018). DNA Structure at the Plasmid Origin-OfTransfer Indicates its Potential Transfer Range. Sci. Rep. 8, 1820. doi:10.1038/ s41598-018-20157-y

Zrimec, J., and Lapanje, A. (2015). Fast Prediction of DNA Melting Bubbles Using DNA Thermodynamic Stability. Ieee/acm Trans. Comput. Biol. Bioinform. 12, 1137-1145. doi:10.1109/tcbb.2015.2396057

Zrimec, J. (2020). Multiple Plasmid Origin-Of-Transfer Regions Might Aid the Spread of Antimicrobial Resistance to Human Pathogens. Microbiologyopen 9, e1129. doi:10.1002/mbo3.1129

Zrimec, J. (2020). "Structural Representations of DNA Regulatory Substrates Can Enhance Sequence-Based Algorithms by Associating Functional Sequence Variants," in Proceedings of the 11th ACM International Conference on Bioinformatics, Computational Biology and Health Informatics (Association for Computing Machinery), 1-6.

Zuallaert, J., Godin, F., Kim, M., Soete, A., Saeys, Y., and De Neve, W. (2018). SpliceRover: Interpretable Convolutional Neural Networks for Improved Splice Site Prediction. Bioinformatics 34, 4180-4188. doi:10.1093/bioinformatics/bty497

Conflict of Interest: The authors declare that the research was conducted in the absence of any commercial or financial relationships that could be construed as a potential conflict of interest.

Copyright (๑) 2021 Zrimec, Buric, Kokina, Garcia and Zelezniak. This is an open-access article distributed under the terms of the Creative Commons Attribution License (CC BY). The use, distribution or reproduction in other forums is permitted, provided the original author(s) and the copyright owner(s) are credited and that the original publication in this journal is cited, in accordance with accepted academic practice. No use, distribution or reproduction is permitted which does not comply with these terms. 